Sharif University of Technology
Scientia Iranica
Transactions E: Industrial Engineering
h.te://scientiairanica.sharif.edu
IRAN ICA

\title{
Bonferroni harmonic mean operators based on two-dimensional uncertain linguistic information and their applications in land utilization ratio evaluation
}

\author{
P. Liu* and W. Liu \\ School of Management Science and Engineering, Shandong University of Finance and Economics, Jinan 250014, Shandong, China.
}

Received 16 January 2017; received in revised form 13 March 2017; accepted 30 April 2018

\section{KEYWORDS \\ 2-dimension uncertain linguistic; \\ Weighted Bonferroni \\ harmonic mean; \\ Multi-attribute group \\ decision making.}

\begin{abstract}
The Bonferroni Mean (BM) enjoys the advantage of capturing the interrelationship among the input arguments, and the harmonic mean is a conservative average lying between the max and min operators. The 2-dimensional uncertain linguistic variables add a subjective evaluation of the reliability of the evaluation results given by decision-makers; therefore, they can express fuzzy information better. In this paper, in order to combine their advantages, the 2-Dimensional Uncertain Linguistic Weighted Bonferroni Mean (2DULWBM) operator is first proposed. However, it cannot consider the case when the given arguments are too high or too low. Therefore, we further propose the 2-Dimensional Uncertain Linguistic Improved Weighted Bonferroni Harmonic Mean (2DULIWBHM) operator, which combines the 2DULWBM with Harmonic Mean. Furthermore, some desirable properties and their special cases are studied. Further, a new method is developed to deal with some Multi-Attribute Group Decision Making (MAGDM) problems under 2-dimensional uncertain linguistic environment based on the proposed operators. Finally, an illustrative example is given to testify the validity of the developed method by comparing it with the other existing methods.
\end{abstract}

(C) 2019 Sharif University of Technology. All rights reserved.

\section{Introduction}

The Multi-Attribute Decision Making (MADM) problems are common in real decision-making. Due to the multiple-attribute decision-making methods proposed by Churchman et al. [1], the classical (i.e., deterministic environment) MADM theory and methods have be developed. However, the attribute values of decisionmaking problems are not always expressed by crisp numbers because of the fuzziness, and some of them are more suitable for expressing by fuzzy information [26], such as fuzzy numbers, linguistic variable [7],

\footnotetext{
*. Corresponding author. Tel.: +86-531-82222188
} E-mail address: Peide.liu@gmail.com (P. Liu)

doi: $10.24200 /$ sci. 2018.20447 intuitionistic fuzzy numbers [8], etc. Particularly, for some qualitative information, we can easily conduct the subjective evaluation by linguistic information. For example, regarding some evaluation problems, such as site-choosing project and vehicle performance, their values can be easily expressed by linguistic terms, such as "very bad," "bad," "general," "good," and "very good". Zadeh [9-11] presented the concept of the linguistic variable firstly, which laid the foundation for the linguistic multiple-attribute decision-making methods; then, it was extended to many different types of fuzzy information, such as uncertain linguistic variables [12-16], 2-dimensional linguistic information [17], etc. Zhu et al. [18] proposed the 2-dimensional linguistic evaluation information to process the linguistic fuzzy decision problems, meaning that decision-makers use both Class I and Class II linguistic information to 
describe their judgment on an object. For example, if a vehicle performance is to be evaluated, Class I linguistic variable is used to perform personal assessment, and Class II linguistic variable is used to evaluate the reliability of assessment results. That is to say, decision-makers can use a 2-dimensional linguistic variable to describe their evaluation of an object. The first variable is used to describe the evaluation result of an attribute given by a decision-maker; the other is used to describe the subjective evaluation of decisionmakers on the reliability of their given results. Liu and Zhang [19] further extended 2-dimensional linguistic information to 2-Dimensional Uncertain Linguistic Information (2DULI) and developed a method to deal with the Multiple Attribute Group Decision Making (MAGDM) problems in which the attribute weight is unknown and attribute values take the form of 2DULI. Then, Liu and Yu [20] extended Power Average (PA) to 2DULI and proposed a 2-dimensional uncertain linguistic power generalized weighted aggregation operator, which could detect the effects of unreasonable data from biased decision-makers. Obviously, 2-Dimensional Uncertain Linguistic Variable (2DULV) can more accurately reflect the evaluation of decision-makers on objects.

The aggregation operators [21-29] have become an important research topic for MADM problems, because they have more advantages than some traditional approaches such as TOPSIS [30], VIKOR [31], ELECTRE [32], TODIM [33], MULTIMOORA [34], and so on. In general, the basic function of the aggregation operators $[8,35]$ is that they can aggregate a set of real values into one. Zhang et al. [36] proposed some power aggregation operators for Intuitionistic Fuzzy Numbers (IFNs), which can consider the influences of unreasonable attribute values based on power weights. Wang et al. [37] proposed some dependent aggregation operators for IFNs, which can eliminate the influences of unreasonable data by dependent weights. Meng et al. [38] proposed the Choquet aggregation operator for IFNs, considering the interaction among aggregating parameters. However, neither of them can consider the relationship between any two attributes. Bonferroni introduced the Bonferroni Mean (BM) operator [39], which can capture the correlations between the input arguments. Further, Wei et al. [40] extended the $\mathrm{BM}$ to the uncertain linguistic variables and proposed an Uncertain Linguistic Bonferroni Mean (ULBM) operator. Liu et al. [41] proposed some intuitionistic fuzzy Dombi Bonferroni mean operators. Ding and Wu [42] proposed some interval-valued hesitant fuzzy Bonferroni mean operators. However, BM cannot consider the case when the given arguments have a great difference; therefore, Sun and Sun [43] combined BM with harmonic operator and proposed Bonferroni Harmonic Mean (BHM), which can be more than adequate to solve the MADM or MAGDM problems by considering the interrelationships and harmonicity.

To sum up the above discussion, the 2Dimensional Uncertain Linguistic Variable (2DULV) can describe qualitative information better, and the BHM operator can consider the interrelationships and harmonicity; therefore, it is meaningful to extend the BHM to the 2DULV. The aim of this paper is to combine the 2DULVs with the BHM operator and to develop the Improved Weighted Bonferroni Harmonic Mean (IWBHM) operator to overcome the weaknesses of the existing operators. Thanks to the advantages of the newly proposed operator, it can capture the interrelationship among the input arguments that have the flexibility by Bonferroni mean parameters, and it can also consider the case when the given arguments have a great difference.

The rest of this paper is organized as follows. In Section 2, the concept and basic operations of 2DULVs, the Bonferroni Mean (BM) operator, 2-Dimensional Uncertain Linguistic Improved Weighted Bonferroni Mean (2DULIWBM) operator, and Improved Weighted Bonferroni Harmonic Mean (IWBHM) operators are briefly reviewed. In Section 3, the 2-Dimensional Uncertain Linguistic Improved Weighted Bonferroni Harmonic Mean (2DULIWBHM) operator on the basis of the 2DULVs rules is proposed. In Section 4, a decision-making method based on the proposed operators for the MADM problems is proposed in which attribute values take the form of 2-dimensional uncertain linguistic information. In Section 5, an example is used to illustrate the effectiveness of the proposed new method, which is compared with the existing other methods. The conclusions are discussed in Section 6.

\section{Preliminaries}

In this section, some basic concepts including the concept of uncertain linguistic variables, 2DULVs, BM operator, 2-Dimensional Uncertain Linguistic Weighted Bonferroni Mean (2DULWBM) operator, the 2Dimensional Uncertain Linguistic Improved Weighted Bonferroni Mean (2DULIWBM) operator, and Improved Weighted Bonferroni Harmonic Mean (IWBM) operator are introduced.

\subsection{The uncertain linguistic variables}

Suppose that linguistic assessment set $\left[S=\left(s_{\beta} \mid \beta=\right.\right.$ $0,1, \ldots, t-1)$ is a finite and totally ordered discrete terms set, $S_{\beta}$ represents the linguistic variable, and $t$ is the odd number. In practice, $t$ is set to $5,7,9$, etc. For instance, when $t=5$, it is represented as follows: $S=\left(S_{1}, S_{2}, S_{3}, S_{4}\right)=$ (very bad, bad, fair, good, very good).

In order to overcome the loss of information 
during the operational process, discrete linguistic set $S=\left(s_{\beta} \mid \beta=0,1, \ldots, t-1\right)$ is extended to a continuous linguistic set $\tilde{S}=\left(s_{\beta} \mid \beta \in R\right)$ by Xu [44], where $\beta$ is the large enough number, and if $s_{\beta} \in S$, then $s_{\beta}$ is called an original linguistic term, or else $s_{\beta}$ is called an extended linguistic term.

Definition 1 [12]. Suppose that $\tilde{s}=\left[s_{a}, s_{b}\right], s_{a}, s_{b} \in$ $\tilde{S}$, and $0 \leq a \leq b ; S_{a}$ and $S_{b}$ are the lower and upper limits of $\tilde{s}$, respectively, and then $\tilde{s}$ is called an uncertain linguistic variable.

Definition $2[12,44]$. Let $\tilde{S}$ be a set of all uncertain linguistic variables, and $\tilde{s}_{1}=\left[s_{a_{1}}, s_{b_{1}}\right], \tilde{s}_{2}=\left[s_{a_{2}}, s_{b_{2}}\right]$ be any two uncertain linguistic variables and $\lambda \geq 0$; the operation rules are defined as follows:

$$
\begin{aligned}
& \tilde{s}_{1} \oplus \tilde{s}_{2}=\left[s_{a_{1}}, s_{b_{1}}\right] \oplus\left[s_{a_{2}}, s_{b_{2}}\right]=\left[s_{a_{1}+a_{2}}, s_{b_{1}+b_{2}}\right], \\
& \tilde{s}_{1} \otimes \tilde{s}_{2}=\left[s_{a_{1}}, s_{b_{1}}\right] \otimes\left[s_{a_{2}}, s_{b_{2}}\right]=\left[s_{a_{1} \times a_{2}}, s_{b_{1} \times b_{2}}\right], \\
& \lambda \tilde{s}_{1}=\lambda\left[s_{a_{1}}, s_{b_{1}}\right]=\left[s_{\lambda a_{1}}, s_{\lambda b_{1}}\right], \\
& \left(\tilde{s}_{1}\right)^{\lambda}=\left[s_{\left(a_{1}\right)^{\lambda}}, s_{\left(b_{1}\right)^{\lambda}}\right] .
\end{aligned}
$$

However, the uncertain linguistic variable is just used to describe linguistic evaluation result of an attribute given by a decision-maker. It cannot describe the subjective evaluation of decision-makers on the reliability of their given results. To solve this problem, the 2DULV was proposed by Liu and Zhang [19].

\subsection{The 2-Dimensional Uncertain Linguistic Variable (2DULV)}

Definition 3 [20]. Let $\hat{s}_{1}=\left(\left[\dot{s}_{a}, \dot{s}_{b}\right]\left[\ddot{s}_{c}, \ddot{s}_{d}\right]\right)$, where $\left[\dot{s}_{a}, \dot{s}_{b}\right]$ is Class I uncertain linguistic variable, which represents decision-maker's judgment on an evaluated object, $\dot{s}_{a}$ and $\dot{s}_{b}$, are the elements from predefined linguistic evaluation set $S_{I}=\left(\dot{s}_{0}, \dot{s}_{1}, \ldots, \dot{s}_{t-1}\right)$, while $\left[\ddot{s}_{c}, \ddot{s}_{d}\right]$ is Class II uncertain linguistic variable which represents the reliability of the subjective evaluation of their given results, $\ddot{s}_{c}$ and $\ddot{s}_{d}$, are the elements from the predefined linguistic evaluation set $S_{I I}=$ $\left(\dot{s}_{0}, \dot{s}_{1}, \ldots, \dot{s}_{L-1}\right) ;$ then, $\hat{s}$ is called the 2-dimensional uncertain linguistic variable.

In order to reduce the loss of linguistic information as well as linguistic variable, the discrete linguistic assessment sets of 2-dimensional uncertain linguistic information are extended to continuous linguistic assessment sets, such that $\dot{s}_{a}, \dot{s}_{b} \in \tilde{S}_{I}=\left(\dot{s}_{\beta} \mid \beta \in[0, l]\right)$ and $\ddot{s}_{c}, \ddot{s}_{d} \in \tilde{S}_{I I}=\left\{\ddot{s}_{\beta} \mid \beta \in\left[0, l^{\prime}\right]\right\}$, where $l$ and $l^{\prime}$ are the two sufficiently large numbers. At the same time, $\hat{S}$ is expressed as the set of all 2-dimensional uncertain linguistic variables.

\subsection{The operational rules and characteristics of $2 D U L V s$}

Definition 4 [20]. Let $\hat{s}_{1}=\left(\left[\dot{s}_{a_{1}}, \dot{s}_{b_{1}}\right]\left[\ddot{s}_{c_{1}}, \ddot{s}_{d_{1}}\right]\right)$ and $\hat{s}_{2}=\left(\left[\dot{s}_{a_{2}}, \dot{s}_{b_{2}}\right]\left[\ddot{s}_{c_{2}}, \ddot{s}_{d_{2}}\right]\right)$ be any two 2DULVs and $\lambda \geq$ 0 . Then, the operational rules of $2 \mathrm{DULV}$ s are defined as follows:

$$
\begin{aligned}
& \hat{s}_{1} \oplus \hat{s}_{2}=\left(\left[\dot{s}_{a_{1}+a_{2}}, \dot{s}_{b_{1}+b_{2}}\right]\left[\ddot{s}_{\min \left(c_{1}, c_{2}\right)}, \ddot{s}_{\min \left(d_{1}, d_{2}\right)}\right]\right), \\
& \hat{s}_{1} \otimes \hat{s}_{2}=\left(\left[\dot{s}_{a_{1} \times a_{2}}, \dot{s}_{b_{1} \times b_{2}}\right]\left[\ddot{s}_{\min \left(c_{1}, c_{2}\right)}, \ddot{s}_{\min \left(d_{1}, d_{2}\right)}\right]\right), \\
& \lambda \hat{s}_{1}=\left(\left[\dot{s}_{\lambda \times a_{1}}, \dot{s}_{\lambda \times b_{1}}\right]\left[\ddot{s}_{c_{1}}, \ddot{s}_{d_{1}}\right]\right) \lambda \geq 0, \\
& \left(\hat{s}_{1}\right)^{\lambda}=\left(\left[\dot{s}_{\left(a_{1}\right)^{\lambda}}, \dot{s}_{\left(b_{1}\right)^{\lambda}}\right]\left[\ddot{s}_{c_{1}}, \ddot{s}_{d_{1}}\right]\right) .
\end{aligned}
$$

When $\lambda<0$, the operational rule (4) is defined as follows:

$$
\left(\hat{s}_{1}\right)^{\lambda}=\left(\left[\dot{s}_{\left(b_{1}\right)^{\lambda}}, \dot{s}_{\left(a_{1}\right)^{\lambda}}\right]\left[\ddot{s}_{c_{1}}, \ddot{s}_{d_{1}}\right]\right) .
$$

Theorem 1 [20]. Let $\hat{s}_{1}=\left(\left[\dot{s}_{a_{1}}, \dot{s}_{b_{1}}\right]\left[\ddot{s}_{c_{1}}, \ddot{s}_{d_{1}}\right]\right), \hat{s}_{2}=$ $\left(\left[\dot{s}_{a_{2}}, \dot{s}_{b_{2}}\right]\left[\ddot{s}_{c_{2}}, \ddot{s}_{d_{2}}\right]\right)$, and $\hat{s}_{3}=\left(\left[\dot{s}_{a_{3}}, \dot{s}_{b_{3}}\right]\left[\ddot{s}_{c_{3}}, \ddot{s}_{d_{3}}\right]\right)$ be three 2DULVs, and $\lambda, \lambda_{1}$, and $\lambda_{2} \geq 0$. Then:

$$
\begin{aligned}
& \hat{s}_{1} \oplus \hat{s}_{2}=\hat{s}_{2} \oplus \hat{s}_{1}, \\
& \hat{s}_{1} \otimes \hat{s}_{2}=\hat{s}_{2} \otimes \hat{s}_{1}, \\
& \hat{s}_{1} \oplus \hat{s}_{2} \oplus \hat{s}_{3}=\hat{s}_{1} \oplus\left(\hat{s}_{2} \oplus \hat{s}_{3}\right), \\
& \hat{s}_{1} \otimes \hat{s}_{2} \otimes \hat{s}_{3}=\hat{s}_{1} \otimes\left(\hat{s}_{2} \otimes \hat{s}_{3}\right), \\
& \hat{s}_{1} \otimes\left(\hat{s}_{2} \oplus \hat{s}_{3}\right)=\left(\hat{s}_{1} \otimes \hat{s}_{2}\right) \oplus\left(\hat{s}_{1} \otimes \hat{s}_{3}\right), \\
& \lambda\left(\hat{s}_{1} \oplus \hat{s}_{2}\right)=\left(\lambda \hat{s}_{1}\right) \oplus\left(\lambda \hat{s}_{2}\right), \\
& \left(\lambda_{1}+\lambda_{2}\right) \hat{s}_{1}=\left(\lambda_{1} \hat{s}_{1}\right) \oplus\left(\lambda_{2} \hat{s}_{1}\right) .
\end{aligned}
$$

Definition 5 [20]. Let $\hat{s}_{1}=\left(\left[\dot{s}_{a_{1}}, \dot{s}_{b_{1}}\right]\left[\ddot{s}_{c_{1}}, \ddot{s}_{d_{1}}\right]\right)$ be a $2 \mathrm{DULV}$. Then, the expected value $E\left(\hat{s}_{1}\right)$ of $\hat{s}_{1}$ is defined as follows:

$E\left(\hat{s}_{1}\right)=\frac{a_{1}+b_{1}}{2 \times(t-1)} \times \frac{c_{1}+d_{1}}{2 \times(L-1)}$,

where $t$ and $L$ can be defined in Definition 3 .

Let $\hat{s}_{1}=\left(\left[\dot{s}_{a_{1}}, \dot{s}_{b_{1}}\right]\left[\ddot{s}_{c_{1}}, \ddot{s}_{d_{1}}\right]\right)$ and $\hat{s}_{2}=\left(\left[\dot{s}_{a_{2}}, \dot{s}_{b_{2}}\right]\right.$ $\left.\left[\ddot{s}_{c_{2}}, \ddot{s}_{d_{2}}\right]\right)$ be any two 2DULVs. If $E\left(\hat{s}_{1}\right) \geq E\left(\hat{s}_{2}\right)$, then $\hat{s}_{1} \geq \hat{s}_{2}$, or vice versa.

Example 1. If $t=7, L=5$, and $\hat{s}_{1}=\left(\left[\dot{s}_{4}, \dot{s}_{5}\right]\left[\ddot{s}_{2}\right.\right.$, $\left.\left.\ddot{s}_{3}\right]\right), \hat{s}_{2}=\left(\left[\dot{s}_{3}, \dot{s}_{4}\right]\left[\ddot{s}_{1}, \ddot{s}_{2}\right]\right)$, then:

$E\left(\hat{s}_{1}\right)=\frac{4+5}{2 \times 6} \times \frac{2+3}{2 \times 4}=\frac{15}{32}$,
$E\left(\hat{s}_{2}\right)=\frac{3+4}{2 \times 6} \times \frac{1+2}{2 \times 4}=\frac{7}{32}$.

Therefore, we have $\hat{s}_{1}>\hat{s}_{2}$. 


\subsection{BM and 2DULIWBM operators}

Definition 6 [39]. Let $p, q \geq 0$ and $\tilde{a}_{i}(i=1,2, \cdots$, $n$ ) be a collection of non-negative numbers. If:

$B M^{p, q}\left(\tilde{a}_{1}, \tilde{a}_{2}, \cdots, \tilde{a}_{n}\right)=\left(\frac{1}{n(n-1)} \sum_{\substack{i, j=1 \\ i \neq j}}^{n} \tilde{a}_{i}^{p} \tilde{a}_{j}^{q}\right)^{\frac{1}{p+q}}$

then $B M^{p, q}$ is called the Bonferroni Mean (BM).

Obviously, the BM has the following properties:

1. Property 1 (Idempotency): $B M^{p, q}(\tilde{a}, \tilde{a}, \cdots, \tilde{a})=\tilde{a}$.

2. Property 2 (Monotonicity): if $\tilde{a}_{i} \geq \tilde{b}_{i}(i=1,2$, $\ldots, n)$ then $B M^{p, q}\left(\tilde{a}_{1}, \tilde{a}_{2}, \cdots, \tilde{a}_{n}\right) \geq B M^{p, q}\left(\tilde{b}_{1}, \tilde{b}_{2}\right.$, $\left.\cdots, \tilde{b}_{n}\right)$.

3. Property 3 (Boundedness): $\min _{i}\left\{\tilde{a}_{i}\right\} \leq B M^{p, q}\left(\tilde{a}_{1}\right.$, $\left.\tilde{a}_{2}, \cdots, \tilde{a}_{n}\right) \leq \max _{i}\left\{\tilde{a}_{i}\right\}$.

The Bonferroni Mean (BM) operator can consider the interrelationships between the aggregated parameters. To consider the advantages of $2 \mathrm{DULV}$ and BM, the 2-Dimensional Uncertain Linguistic Weighted Bonferroni Mean (2DULWBM) is proposed.

Definition 7. Let $p, q \geq 0$, and:

$$
\hat{s}_{i}=\left(\left[\dot{s}_{a_{i}}, \dot{s}_{b_{i}}\right]\left[\ddot{s}_{c_{i}}, \ddot{s}_{d_{i}}\right]\right) \quad(i=1,2, \ldots, n)
$$

be a collection of 2 DULVs with the weight vector:

$$
w=\left(w_{1}, w_{2}, \cdots, w_{n}\right)^{T}
$$

such that $w_{i} \geq 0(i=1,2, \cdots, n)$ and $\sum_{i=1}^{n} w_{i}=1$. Then, the 2-dimensional uncertain linguistic weighted Bonferroni mean operator can be defined as follows:

$\operatorname{2DULWB} M^{p, q}\left(\hat{s}_{1}, \hat{s}_{2}, \cdots, \hat{s}_{n}\right)$

$$
=\left(\bigoplus_{\substack{i, j=1 \\ i \neq j}}^{n}\left(\left(n w_{i} \hat{s}_{i}^{p}\right) \otimes\left(n w_{j} \hat{s}_{j}^{q}\right)\right)\right)^{\frac{1}{p+q}}
$$

It is obvious that Eq. (19) does not have the idempotency; thus, the improved 2-Dimensional Uncertain Linguistic Weighted Bonferroni Mean (2DULIWBM) operator is proposed as follows:

$$
\begin{aligned}
& \operatorname{2DULIWB} M^{p, q}\left(\hat{s}_{1}, \hat{s}_{2}, \cdots, \hat{s}_{n}\right) \\
& =\frac{1}{\left(\sum_{\substack{i, j=1 \\
i \neq j}}^{n}\left(w_{i} w_{j}\right)\right)^{\frac{1}{p+q}}}\left(\bigoplus_{\substack{i, j=1 \\
i \neq j}}^{n}\left(\left(w_{i} \hat{s}_{i}^{p}\right) \otimes\left(w_{j} \hat{s}_{j}^{q}\right)\right)\right)_{(20)}^{\frac{1}{p+q}} .
\end{aligned}
$$

Based on the operational laws of the 2DULVs, the results are derived, as shown in Theorem 2 .

Theorem 2. Let $p, q \geq 0$, and:

$$
\hat{s}_{i}=\left(\left[\dot{s}_{a_{i}}, \dot{s}_{b_{i}}\right]\left[\ddot{s}_{c_{i}}, \ddot{s}_{d_{i}}\right]\right) \quad(i=1,2, \ldots, n),
$$

be a collection of $2 \mathrm{DULV}$ s with the weight vector $w=\left(w_{1}, w_{2}, \cdots, w_{n}\right)^{T}$ such that $w_{i} \geq 0(i=$ $1,2, \cdots, n)$ and $\sum_{i=1}^{n} w_{i}=1$; then, the result aggregated from Eq. (20) is still an 2DULV, and is calculated by Eq. (21) as shown in Box I.

Proof. On the basis of Definition 4, the aggregated value is also a 2 DULV. Therefore, we can prove Eq. (21) by using a mathematical induction on $n$.

Firstly, we need to prove Eq. (22) shown in Box II.

According to the operations of $2 \mathrm{DULV}$ defined in Eqs. (6)-(8), we have:

$$
\begin{aligned}
w_{i} \hat{s}_{i}^{p} & =\left(\left[\dot{s}_{w_{i} a_{i} p}, \dot{s}_{w_{i} b_{i} p}\right]\left[\ddot{s}_{c_{i}}, \ddot{s}_{d_{i}}\right]\right), \\
w_{j} \hat{s}_{j}^{q} & =\left(\left[\dot{s}_{w_{j} a_{j}}, \dot{s}_{w_{j} b_{j} q}\right]\left[\ddot{s}_{c_{j}}, \ddot{s}_{d_{j}}\right]\right),
\end{aligned}
$$

$$
\begin{aligned}
& 2 D U L I W B M^{p, q}\left(\hat{s}_{1}, \hat{s}_{2}, \cdots, \hat{s}_{n}\right)
\end{aligned}
$$

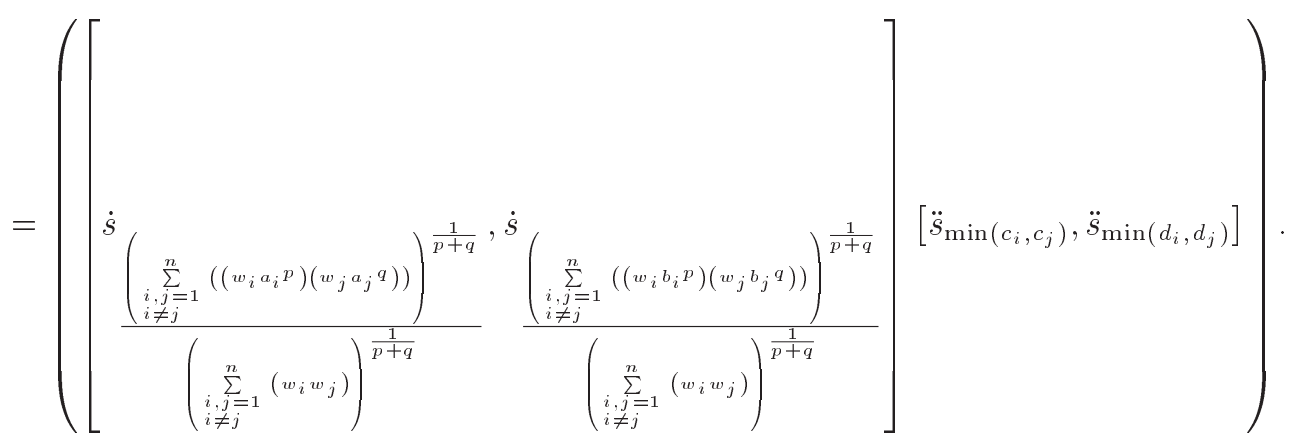




$$
\bigoplus_{\substack{i, j=1 \\ i \neq j}}^{n}\left(\left(w_{i} \hat{s}_{i}^{p}\right) \otimes\left(w_{j} \hat{s}_{j}^{q}\right)\right)=\left(\left[s\left(\sum_{\substack{i, j=1 \\ i \neq j}}^{n}\left(\left(w_{i} a_{i}^{p}\right)\left(w_{j} a_{j}^{q}\right)\right)\right), s\left(\sum_{\substack{i, j=1 \\ i \neq j}}^{n}\left(\left(w_{i} b_{i}^{p}\right)\left(w_{j} b_{j}^{q}\right)\right)\right)\right],\left[\ddot{s}_{\min \left(c_{i}, c_{j}\right)}, \ddot{s}_{\min \left(d_{i}, d_{j}\right)}\right)\right] .
$$

Box II

$$
\begin{aligned}
\left(w_{i} \hat{s}_{i}^{p}\right) \otimes\left(w_{j} \hat{s}_{j}^{q}\right) & \\
= & \left(\left[\dot{s}_{\left(w_{i} a_{i} p\right)\left(w_{j} a_{j}^{q}\right)}, \dot{s}_{\left(w_{i} b_{i} p\right)\left(w_{j} b_{j}^{q}\right)}\right]\right. \\
& \left.\ddot{s}_{\min \left(c_{i}, c_{j}\right)}, \ddot{s}_{\min \left(d_{i}, d_{j}\right)}\right) .
\end{aligned}
$$

When $n=2$, by Eqs. (5) and (24), we can obtain:

$$
\begin{aligned}
& \underset{\substack{i, j=1 \\
i \neq j}}{\stackrel{\oplus}{i \neq j}}\left(\left(w_{i} \hat{s}_{i}^{p}\right) \otimes\left(w_{j} \hat{s}_{j}^{q}\right)\right)=\left(w_{1} \hat{s}_{1}^{p}\right) \otimes\left(w_{2} \hat{s}_{2}^{q}\right) \\
& +\left(w_{2} \hat{s}_{2}^{p}\right) \otimes\left(w_{1} \hat{s}_{1}^{q}\right) \\
& =\left(\left[s\left(\left(w_{1} a_{1}{ }^{p}\right)\left(w_{2} a_{2} q\right)+\left(w_{2} a_{2}^{p}\right)\left(w_{1} a_{1}^{q}\right)\right),\right.\right. \\
& s_{\left.\left(\left(w_{1} b_{1}^{p}\right)\left(w_{2} b_{2}^{q}\right)+\left(w_{2} b_{2}^{p}\right)\left(w_{1} b_{1}^{q}\right)\right)\right],} \\
& \left.\left[\ddot{s}_{\min \left(c_{1}, c_{2}\right)}, \ddot{s}_{\min \left(d_{1}, d_{2}\right)}\right]\right),
\end{aligned}
$$

that is, when $n=2$, Eq. (22) is right.

If Eq. (22) holds for $n=K$, then we have Eq. (25) shown in Box III.

When $n=K+1$, we have:

$$
\begin{aligned}
& \underset{\substack{i, j=1 \\
i \neq j}}{K+1}\left(\left(w_{i} \hat{s}_{i}^{p}\right) \otimes\left(w_{j} \hat{s}_{j}^{q}\right)\right)=\underset{\substack{i, j=1 \\
i \neq j}}{\stackrel{K}{i}}\left(\left(w_{i} \hat{s}_{i}^{p}\right)\right. \\
& \left.\otimes\left(w_{j} \hat{s}_{j}^{q}\right)\right)+\underset{i=1}{K}\left(\left(w_{i} \hat{s}_{i}^{p}\right)\right. \\
& \left.\otimes\left(w_{K+1} \hat{s}_{K+1}^{q}\right)\right)+\underset{j=1}{K}\left(\left(w_{K+1} \hat{s}_{K+1}^{p}\right) \otimes\left(w_{j} \hat{s}_{j}^{q}\right)\right) .
\end{aligned}
$$

Firstly, we prove Eq. (27) shown in Box IV. The mathematical induction on $K$ is used as follows.

(a) When $K=2$, we get:

$$
\begin{aligned}
& \left(w_{i} \hat{s}_{i}^{p}\right) \otimes\left(w_{3} \hat{s}_{3}^{q}\right) \\
& =\left(\left[s_{\left(\left(w_{i} a_{i}^{p}\right)\left(w_{3} a_{3}^{q}\right)\right)}, s_{\left.\left(\left(w_{i} b_{i}^{p}\right)\left(w_{3} b_{3}^{q}\right)\right)\right],}\right.\right. \\
& \left.\left[\ddot{s}_{\min \left(c_{i}, c_{3}\right)}, \ddot{s}_{\min \left(d_{i}, d_{3}\right)}\right]\right) \underset{i=1}{\stackrel{2}{\oplus}}\left(\left(w_{i} \hat{s}_{i}^{p}\right)\right. \\
& \left.\otimes\left(w_{K+1} \hat{s}_{K+1}^{q}\right)\right)=\left(w_{1} \hat{s}_{1}^{p}\right) \otimes\left(w_{3} \hat{s}_{3}^{q}\right) \\
& +\left(w_{2} \hat{s}_{2}^{p}\right) \otimes\left(w_{3} \hat{s}_{3}^{q}\right) \\
& =\left(\left[s\left(\left(w_{1} a_{1} p\right)\left(w_{3} a_{3}{ }^{q}\right)+\left(w_{2} a_{2}^{p}\right)\left(w_{3} a_{3}^{q}\right)\right),\right.\right.
\end{aligned}
$$

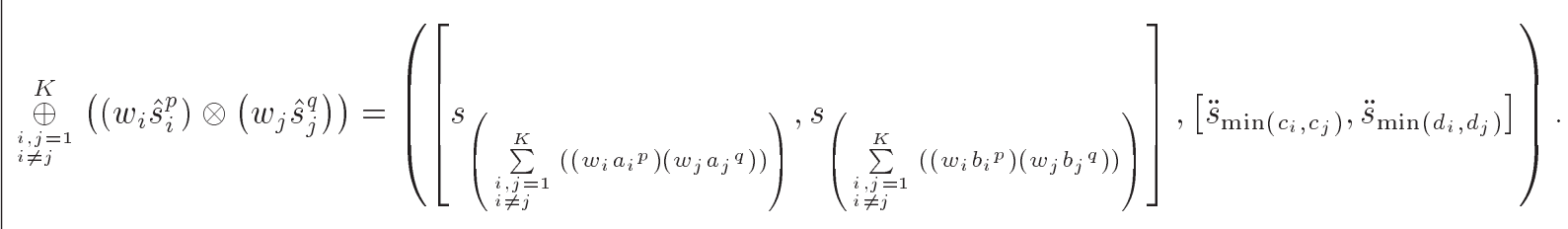

Box III

$$
\bigoplus_{i=1}^{K}\left(\left(w_{i} \hat{s}_{i}^{p}\right) \otimes\left(w_{K+1} \hat{s}_{K+1}^{q}\right)\right)=\left(\left[s\left(\sum_{i=1}^{K}\left(w_{i} a_{i}^{p}\right)\left(w_{K+1} a_{K+1} q\right)\right)^{, s}\left(\sum_{i=1}^{K}\left(w_{i} b_{i}^{p}\right)\left(w_{K+1} b_{K+1} q\right)\right)\right]\left[\ddot{s}_{\min \left(c_{i}, c_{K+1}\right)}, \ddot{s}_{\min \left(d_{i}, d_{K+1}\right)}\right]\right) .
$$




$$
\begin{aligned}
& {\underset{i=1}{\oplus+1}}_{i}\left(\left(w_{i} \hat{s}_{i}^{p}\right) \otimes\left(w_{T+2} \hat{s}_{T+2}^{q}\right)\right)=\bigoplus_{i=1}^{T}\left(\left(w_{i} \hat{s}_{i}^{p}\right) \otimes\left(w_{T+2} \hat{s}_{T+2}^{q}\right)\right)+\left(w_{T+1} \hat{s}_{T+1}^{p}\right) \otimes\left(w_{T+2} \hat{s}_{T+2}^{q}\right) \\
& =\left(\left[s\left(\sum_{i=1}^{T+1}\left(w_{i} a_{i}^{p}\right)\left(w_{T+2} a_{T+2}^{q}\right)\right)^{, s}\left(\sum_{i=1}^{T+1}\left(w_{i} b_{i}^{p}\right)\left(w_{T+2} b_{T+2} q\right)\right)\right]\left[\ddot{s}_{\min \left(c_{i}, c_{T+2}\right)} \ddot{s}_{\min \left(d_{i}, d_{T+2}\right)}\right]\right) .
\end{aligned}
$$

\section{Box $\mathrm{V}$}

$$
\begin{aligned}
& \bigoplus_{j=1}^{K}\left(\left(w_{K+1} \hat{s}_{K+1}^{p}\right) \otimes\left(w_{j} \hat{s}_{j}^{q}\right)\right) \\
& \quad=\left(\left[s\left(\sum_{j=1}^{K}\left(w_{K+1} a_{K+1}^{p}\right)\left(w_{j} a_{j}^{q}\right)\right), s\left(\sum_{j=1}^{K}\left(w_{K+1} b_{K+1}^{p}\right)\left(w_{j} b_{j}^{q}\right)\right)\right]\left[\ddot{s}_{\min \left(c_{K+1}, c_{j}\right)}, \ddot{s}_{\min \left(d_{K+1}, d_{j}\right)}\right]\right) .
\end{aligned}
$$

\section{Box VI}

$$
\begin{aligned}
& \left.s\left(\left(w_{1} b_{1}^{p}\right)\left(w_{3} b_{3}^{q}\right)+\left(w_{2} b_{2}^{p}\right)\left(w_{3} b_{3}^{q}\right)\right)\right], \\
& \left.\left[\ddot{s}_{\min \left(c_{i}, c_{3}\right)}, \ddot{s}_{\min \left(d_{i}, d_{3}\right)}\right]\right) .
\end{aligned}
$$

In other words, when $n=2$, Eq. (27) is right.

(b) If Eq. (27) holds for $K=T$, then:

$$
\begin{aligned}
& \underset{i=1}{\oplus}\left(\left(w_{i} \hat{s}_{i}^{p}\right) \otimes\left(w_{T+1} \hat{s}_{T+1}^{q}\right)\right) \\
& =\left(\left[s\left(\sum_{i=1}^{T}\left(w_{i} a_{i}{ }^{p}\right)\left(w_{T+1} a_{T+1}\right)^{q}\right)\right)^{s}\left(\sum_{i=1}^{T}\left(w_{i} b_{i}^{p}\right)\left(w_{T+1} b_{T+1}{ }^{q}\right)\right)\right] \\
& \left.\left[\ddot{s}_{\min \left(c_{i}, c_{T+1}\right)}, \ddot{s}_{\min \left(d_{i}, d_{T+1}\right)}\right]\right) .
\end{aligned}
$$

When $K=T+1$, we have the relation shown in Box V. In other words, for $K=T+1$, Eq. (27) is also right. Similarly, we can obtain Eq. (28) as shown in Box VI.
Based on Eqs. (25), (27), and (28), we can obtain Eq. (26) as shown in Box VII. Therefore, when $n=$ $K+1$, Eq. (22) is also right, and it is right for all $n$.

Now, it is easy to prove that Eq. (21) is right. By Eq. (22), we obtain the relation shown in Box VIII. Hence, for all $n$, Eq. (21) is true.

Obviously, Eq. (21) has also the properties similar to those of the $B M^{p, q}$ : idempotency, monotonicity, commutativity, and boundedness.

Example 2. Let $\hat{s}_{1}=\left(\left[\dot{s}_{2}, \dot{s}_{3}\right],\left[\ddot{s}_{3}, \ddot{s}_{3}\right]\right), \hat{s}_{2}=\left(\left[\dot{s}_{3}, \dot{s}_{4}\right]\right.$, $\left.\left[\ddot{s}_{2}, \ddot{s}_{3}\right]\right)$, and $\hat{s}_{3}=\left(\left[\dot{s}_{7}, \dot{s}_{9}\right],\left[\ddot{s}_{2}, \ddot{s}_{2}\right]\right)$ be three 2DULVs (suppose that Class I linguistic set is $S_{I}=\left(\dot{s}_{0}, \dot{s}_{1}, \ldots\right.$, $\left.\dot{s}_{9}\right)$ and Class II linguistic set is $S_{I I}=\left(\dot{s}_{0}, \dot{s}_{1}, \dot{s}_{2}\right.$, $\left.\left.\dot{s}_{3}, \dot{s}_{4}\right)\right)$, and $w=(0.4,0.25,0.35)^{T}$ be the weight vector of $\hat{s}_{i}(i=1,2,3)$. Then, the operator 2DULIWBM can be used to aggregate three 2DULVs as follows.

The comprehensive value $\hat{s}=\left(\left[\dot{s}_{a}, \dot{s}_{b}\right]\left[\ddot{s}_{c}, \ddot{s}_{d}\right]\right)$ can be calculated by Eq. (21) (without loss of generality, we suppose that $p=q=1$ ), and we obtain the relation shown in Box IX.

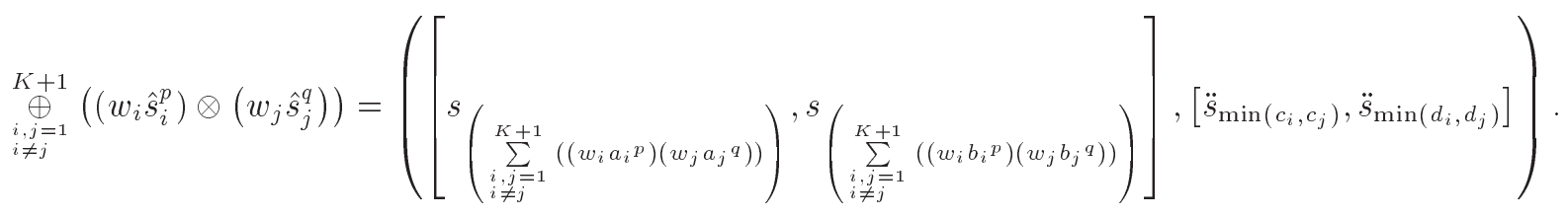




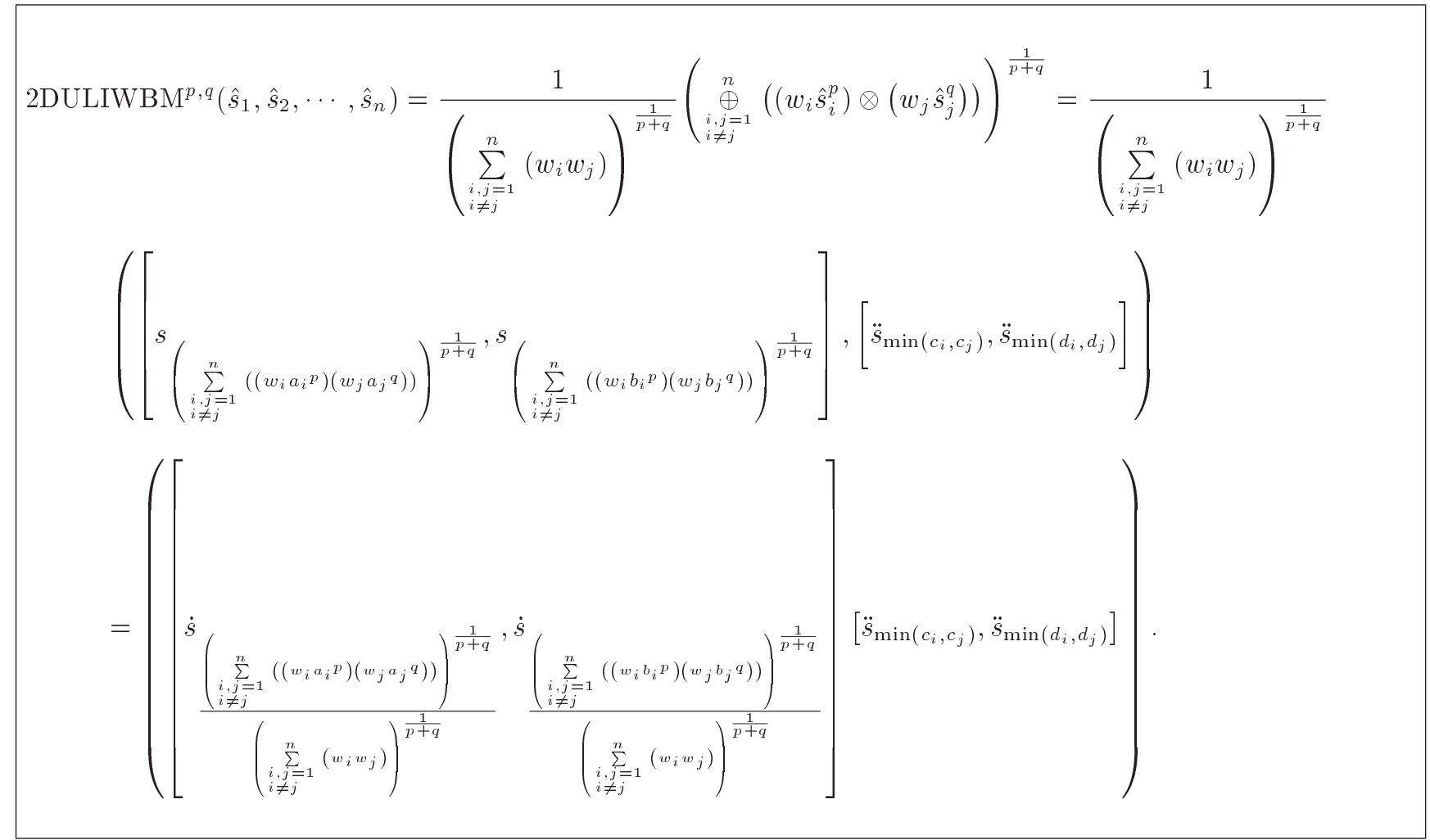

Box VIII

$\operatorname{DDULIWBM}^{1,1}\left(\hat{s}_{1}, \hat{s}_{2}, \hat{s}_{3}\right)$

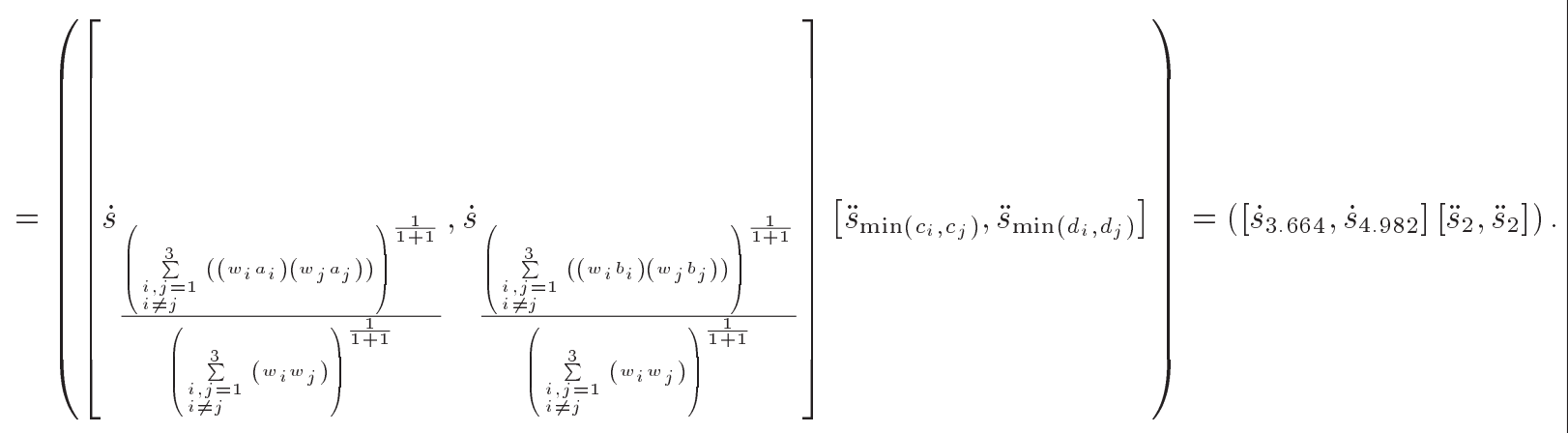

Box IX

However, BM operator cannot relieve the effects when the given arguments are too high or too low. Then, Sun and Sun [43] proposed Weighted Bonferroni Harmonic Mean (WBHM) operator to solve this problem.

\subsection{Improved Weighted Bonferroni Harmonic Mean (IWBHM) operator}

The Weighted Bonferroni Harmonic Mean (WBHM) operator was proposed by Sun and Sun [43], and it is combined by Bonferroni Mean (BM) [39] and Harmonic Mean (HM) [45], which is defined as follows.

Definition 8 [43]. Let $p, q \geq 0$ and $\tilde{a}_{i}(i=$ $1,2, \cdots, n)$ be a collection of nonnegative numbers with the weight vector $w=\left(w_{1}, w_{2}, \cdots, w_{n}\right)^{T}$ such that $w_{i} \geq 0(i=1,2, \cdots, n)$ and $\sum_{i=1}^{n} w_{i}=1$. The WBHM is defined as follows: 


$$
W B H M^{p, q}\left(\tilde{a}_{1}, \tilde{a}_{2}, \cdots, \tilde{a}_{n}\right)=\frac{1}{\left(\sum_{\substack{i, j=1 \\ i \neq j}}^{n} \frac{w_{i} w_{j}}{\tilde{a}_{i}^{p} \tilde{a}_{j}^{j}}\right)^{\frac{1}{p+q}}} .
$$

It is obvious that Eq. (29) does not have idempotency; thus, the Improved Weighted Bonferroni Harmonic Mean (IWBHM) operator is proposed as follows.

Definition 9. Let $p, q \geq 0$, and $\tilde{a}_{i}(i=1,2, \cdots, n)$ be a collection of nonnegative numbers with the weight vector, $w=\left(w_{1}, w_{2}, \cdots, w_{n}\right)^{T}$, such that $w_{i} \geq 0(i=$ $1,2, \cdots, n)$ and $\sum_{i=1}^{n} w_{i}=1$. The IWBHM operator is defined as follows:

$\operatorname{IWBHM}^{p, q}\left(\tilde{a}_{1}, \tilde{a}_{2}, \cdots, \tilde{a}_{n}\right)$

$$
=\frac{\left(\sum_{\substack{i, j=1 \\ i \neq j}}^{n}\left(w_{i} w_{j}\right)\right)^{\frac{1}{p+q}}}{\left(\sum_{\substack{i, j=1 \\ i \neq j}}^{n}\left(\frac{w_{i}}{\tilde{a}_{i}^{i}}\right)\left(\frac{w_{j}}{\tilde{a}_{j}^{q}}\right)\right)^{\frac{1}{p+q}}} .
$$

\section{2-Dimensional Uncertain Linguistic Improved Weighted Bonferroni Harmonic Mean (2DULIWBHM) operator}

In this section, based on the operational rules of 2DULV and the improved weighted Bonferroni harmonic mean, the 2-Dimensional Uncertain Linguistic Improved Weighted Bonferroni Harmonic Mean (2DULIWBHM) operator is proposed; then, some particular cases and properties will be investigated.

Definition 10. Let $\hat{s}_{i}=\left(\left[\dot{s}_{a_{i}}, \dot{s}_{b_{i}}\right]\left[\ddot{s}_{c_{i}}, \ddot{s}_{d_{i}}\right]\right)(i=$ $1,2, \ldots, n)$ be a collection of 2 DULVs with weight vector $w=\left(w_{1}, w_{2}, \cdots, w_{n}\right)^{T}$ such that $w_{i} \geq 0(i=$ $1,2, \cdots, n)$ and $\sum_{i=1}^{n} \omega_{i}=1$; then, the 2-dimensional uncertain linguistic improved weighted Bonferroni harmonic mean operator is defined as follows:

2DULIWBHM $^{p, q}\left(\hat{s}_{1}, \hat{s}_{2}, \cdots, \hat{s}_{n}\right)$

$$
\begin{aligned}
& =\left(\sum_{\substack{i, j=1 \\
i \neq j}}^{n}\left(w_{i} w_{j}\right)\right)^{\frac{1}{p+q}} \\
& \left(\frac{1}{\underbrace{n}_{\substack{i, j=1 \\
i \neq j}}\left(\left(\frac{w_{i}}{\hat{s}_{i}^{i}}\right) \otimes\left(\frac{w_{j}}{\hat{s}_{j}^{q}}\right)\right)}\right)^{\frac{1}{p+q}} .
\end{aligned}
$$

Based on the operational laws of the 2DULVs, the aggregated result from Definition 10 is derived, as shown in Theorem 3.

Theorem 3. Let $p, q \geq 0$, and $\hat{s}_{i}=\left(\left[\dot{s}_{a_{i}}, \dot{s}_{b_{i}}\right]\left[\ddot{s}_{c_{i}}\right.\right.$, $\left.\left.\ddot{s}_{d_{i}}\right]\right)(i=1,2, \ldots, n)$ be a collection of 2 DULVs with the weight vector $w=\left(w_{1}, w_{2}, \cdots, w_{n}\right)^{T}$ such that $w_{i} \geq 0(i=1,2, \cdots, n)$; then, the result aggregated from Eq. (31) is still an 2DULV, and Eq. (32) shown in Box X is obtained.

Proof. Firstly, we can calculate $\hat{s}_{i}^{p}$ and $\hat{s}_{j}^{q}$ and obtain:

$$
\begin{aligned}
& \hat{s}_{i}^{p}=\left(\left[\dot{s}_{a_{i} p}, \dot{s}_{b_{i} p}\right]\left[\ddot{s}_{c_{i}}, \ddot{s}_{d_{i}}\right]\right), \\
& \hat{s}_{j}^{q}=\left(\left[\dot{s}_{a_{j} q}, \dot{s}_{b_{j}}\right]\left[\ddot{s}_{c_{j}}, \ddot{s}_{d_{j}}\right]\right),
\end{aligned}
$$

and they are also 2DULVs. Then, we obtain:

$$
\frac{w_{i}}{\hat{s}_{i}^{p}}=\left(\left[\dot{s}_{\frac{w_{i}}{b_{i}}}, \dot{s}_{\frac{w_{i}}{a_{i}}}\right]\left[\ddot{s}_{c_{i}}, \ddot{s}_{d_{i}}\right]\right) \text {, }
$$$$
\frac{w_{j}}{\hat{s}_{j}^{q}}=\left(\left[\dot{s} \frac{w_{j}}{b_{j}{ }^{q}}, \dot{s}_{\frac{w_{j}}{a_{j}}}\right]\left[\ddot{s}_{c_{j}}, \ddot{s}_{d_{j}}\right]\right),
$$

$$
\begin{aligned}
& \operatorname{2DULIWBHM}^{p, q}\left(\hat{s}_{1}, \hat{s}_{2}, \ldots \hat{s}_{n}\right)
\end{aligned}
$$

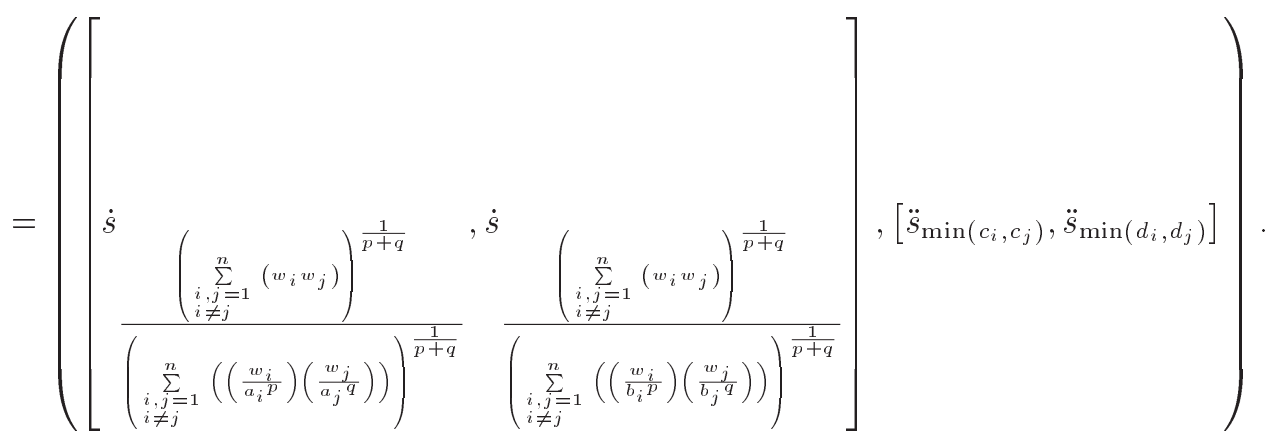


and:

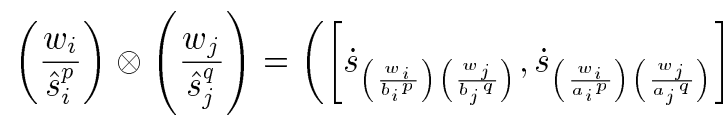

$$
\begin{aligned}
& \left.\left[\ddot{s}_{\min \left(c_{i}, c_{j}\right)}, \ddot{s}_{\min \left(d_{i}, d_{j}\right)}\right]\right) .
\end{aligned}
$$

Then, we obtain the relation shown in Box XI. Therefore, we have the relation shown in Box XII. So, Theorem 3 is proved.

Example 3. If we solve Example 2 according to Theorem 3, then we obtain the relation shown in Box XIII.

Obviously, the result is smaller than Example 2, because our method can eliminate the effect when the given data are too high or too low. In this example, Class I uncertain linguistic variable of $\hat{s}_{3}$ is $\left[\dot{s}_{7}, \dot{s}_{9}\right]$, which is extremely bigger than the other two. The 2DULIWBM operator cannot consider this case; however, the proposed operator in this paper can tackle this shortcoming.

It is easy to prove that the 2DULIWBHM operator has the following properties.
Theorem 4 (Idempotency). If $\hat{s}_{j}=\left(\left[\dot{s}_{a_{j}}, \dot{s}_{b_{j}}\right]\right.$ $\left.\left[\ddot{s}_{c_{j}}, \ddot{s}_{d_{j}}\right]\right) j=1,2 \ldots, n$ all are equal, i.e., $\hat{s}_{j}=\hat{s}=$ $\left(\left[\dot{s}_{a}, \dot{s}_{b}\right]\left[\ddot{s}_{c}, \ddot{s}_{d}\right]\right)$ for all $j$, then:

$\operatorname{2DULIWBHM}^{p, q}(\hat{s}, \hat{s}, \ldots, \hat{s})=\hat{s}$.

Proof. The relation shown in Box XIV completes the proof of Theorem 4.

Theorem 5 (commutativity). If $\left(\hat{s}_{1}^{\prime}, \hat{s}_{2}^{\prime}, \ldots, \hat{s}_{n}^{\prime}\right)$ is any permutation of $\left(\hat{s}_{1}, \hat{s}_{2}, \ldots, \hat{s}_{n}\right)$, then:

$$
\begin{aligned}
& \operatorname{2DULIWBHM}^{p, q}\left(\hat{s}_{1}^{\prime}, \hat{s}_{2}^{\prime}, \ldots, \hat{s}_{n}^{\prime}\right) \\
& \quad=2 \operatorname{DULIWBHM}^{p, q}\left(\hat{s}_{1}, \hat{s}_{2}, \ldots, \hat{s}_{n}\right) .
\end{aligned}
$$

Proof. Based on Eq. (32), we have the relation shown in Box XV. Since $\left(\hat{s}_{1}^{\prime}, \hat{s}_{2}^{\prime}, \ldots ., \hat{s}_{n}^{\prime}\right)$ is any permutation of $\left(\hat{s}_{1}, \hat{s}_{2}, \ldots, \hat{s}_{n}\right)$, then we can get:

$$
\begin{aligned}
& \operatorname{2DULIWBHM}^{p, q}\left(\hat{s}_{1}^{\prime}, \hat{s}_{2}^{\prime}, \ldots ., \hat{s}_{n}^{\prime}\right) \\
& \quad=2 \operatorname{DULIWBHM}^{p, q}\left(\hat{s}_{1}, \hat{s}_{2}, \ldots ., \hat{s}_{n}\right) .
\end{aligned}
$$

Theorem 6 (monotonicity). Let:

$$
\hat{s}_{\eta}=\left(\left[\dot{s}_{a_{\eta}}, \dot{s}_{b_{\eta}}\right],\left[\ddot{s}_{c_{\eta}}, \ddot{s}_{d_{\eta}}\right]\right)
$$

and:

$$
\left(\frac{1}{\bigoplus_{\substack{i, j=1 \\ i \neq j}}^{n}\left(\left(\frac{w_{i}}{\hat{s}_{i}^{p}}\right) \otimes\left(\frac{w_{j}}{\hat{s}_{j}^{q}}\right)\right)}\right)^{\frac{1}{p+q}}=\left(\left[\dot{s} \frac{1}{\left(\sum_{\substack{i, j=1 \\ i \neq j}}^{n}\left(\left(\frac{w_{i}}{a_{i}}\right)\left(\frac{w_{j}}{a_{j}}\right)\right)\right)^{\frac{1}{p+q}}}, \dot{s} \frac{1}{\left(\sum_{\substack{i, j=1 \\ i \neq j}}^{n}\left(\left(\frac{w_{i}}{b_{i}}\right)\left(\frac{w_{j}}{b_{j}}\right)\right)\right)^{\frac{1}{p+q}}}\right]\left[\ddot{s}_{\left.\min \left(c_{i}, c_{j}\right), \ddot{s}_{\min }\left(d_{i}, d_{j}\right)\right]}\right]\right.
$$

$\left(\sum_{\substack{i, j=1 \\ i \neq j}}^{n}\left(w_{i} w_{j}\right)\right)^{\frac{1}{p+q}}\left(\frac{1}{\bigoplus_{\substack{i, j=1 \\ i \neq j}}^{n}\left(\left(\frac{w_{i}}{\hat{s}_{i}^{p}}\right) \otimes\left(\frac{w_{j}}{\hat{s}_{j}^{q}}\right)\right)}\right)^{\frac{1}{p+q}}$

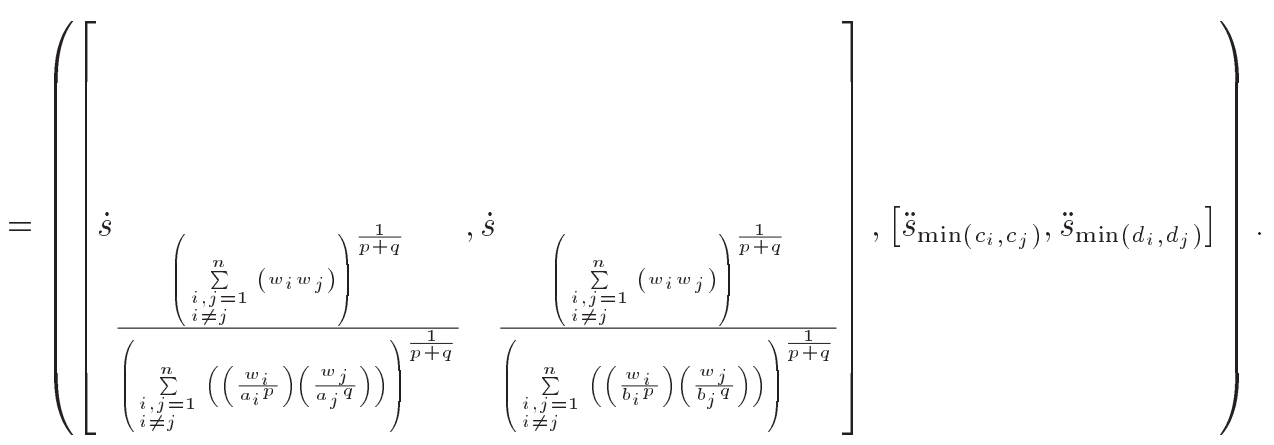




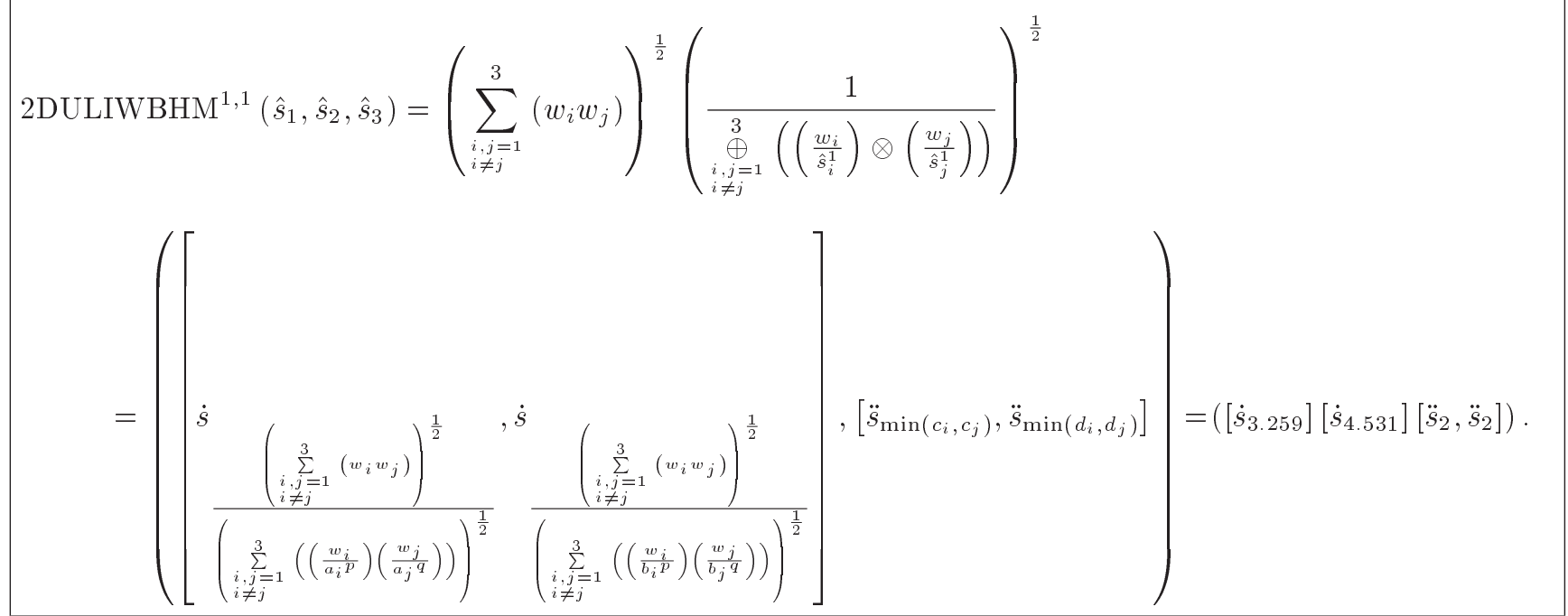

Box XIII

$\operatorname{DDULIWBHM}^{p, q}(\hat{s}, \hat{s}, \ldots ., \hat{s})$

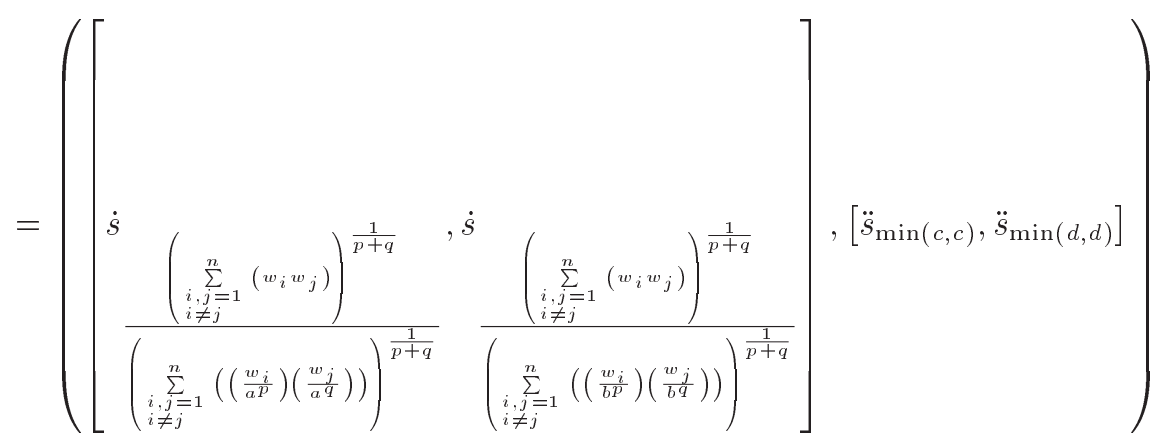

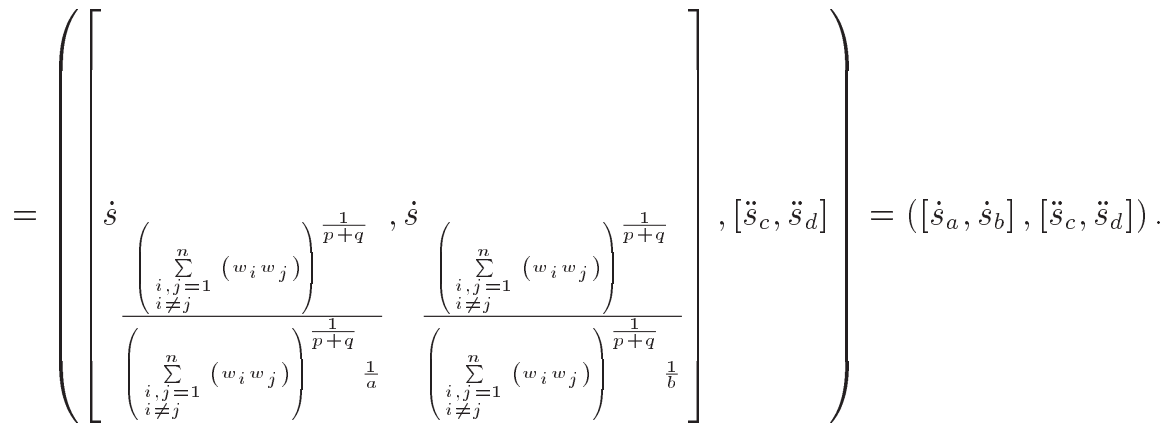

Box XIV

$$
\begin{gathered}
\hat{s}_{\eta}^{\prime}=\left(\left[\dot{s}_{a^{\prime} \eta}, \dot{s}_{b^{\prime} \eta}\right],\left[\ddot{s}_{c^{\prime} \eta}, \ddot{s}_{d^{\prime} \eta}\right]\right) \\
(\eta=1,2, \ldots, n)
\end{gathered}
$$

be any two collections of 2DULVs. If $a_{\eta} \geq a_{\eta}^{\prime}, b_{\eta} \geq b_{\eta}^{\prime}$, $c_{\eta} \geq c_{\eta}^{\prime}$, and $d_{\eta} \geq d_{\eta}^{\prime}$ for all $\eta$, then:

$\operatorname{DDULIWBHM}^{p, q}\left(\hat{s}_{1}, \hat{s}_{2}, \ldots, \hat{s}_{n}\right)$

$$
\geq \operatorname{DDULIWBHM}^{p, q}\left(\hat{s}_{1}^{\prime}, \hat{s}_{2}^{\prime}, \ldots ., \hat{s}_{n}^{\prime}\right) .
$$

Proof. Since $a_{\eta} \geq a_{\eta}^{\prime}, b_{\eta} \geq b_{\eta}^{\prime}, c_{\eta} \geq c_{\eta}^{\prime}, d_{\eta} \geq d_{\eta}^{\prime}$, and $p, q>0$, then based on Eq. (32), we have the relation shown in Box XVI.

Since $a_{\eta} \geq a_{\eta}^{\prime}$, we get:

$$
\left(\sum_{\substack{i, j=1 \\ i \neq j}}^{n}\left(\left(\frac{w_{i}}{a_{i} p}\right)\left(\frac{w_{j}}{a_{j} q}\right)\right)\right)^{\frac{1}{p+q}}
$$


$2 \operatorname{DULIWBHM}^{p, q}\left(\hat{s}_{1}, \hat{s}_{2}, \cdots, \hat{s}_{n}\right)$

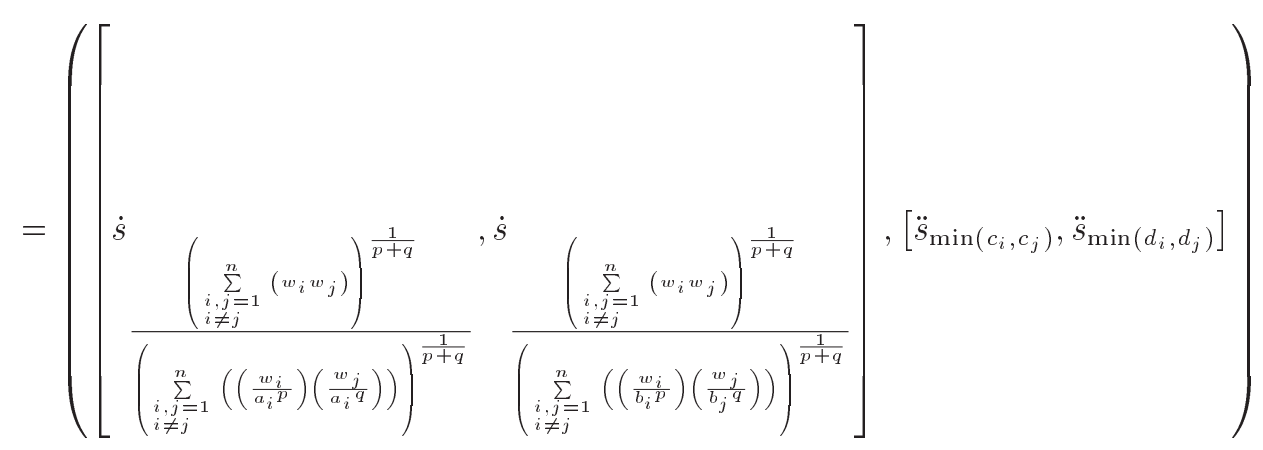

$2 \operatorname{DULIWBHM}^{p, q}\left(\hat{s}_{1}^{\prime}, \hat{s}_{2}^{\prime}, \cdots, \hat{s}_{n}^{\prime}\right)$

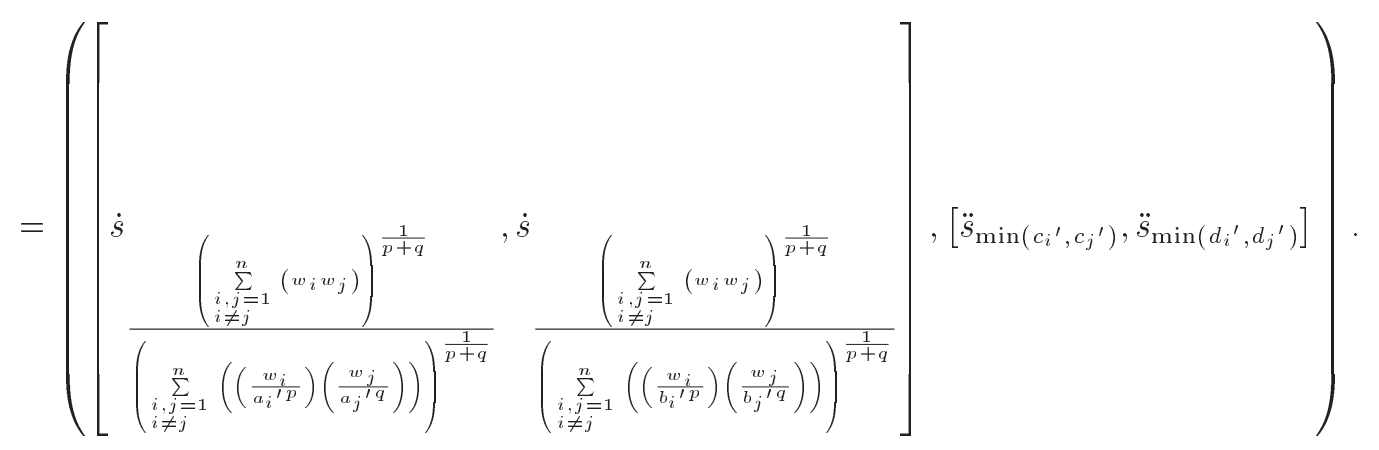

Box XV

$2 \operatorname{DULIWBHM}^{p, q}\left(\hat{s}_{1}, \hat{s}_{2}, \cdots, \hat{s}_{n}\right)$

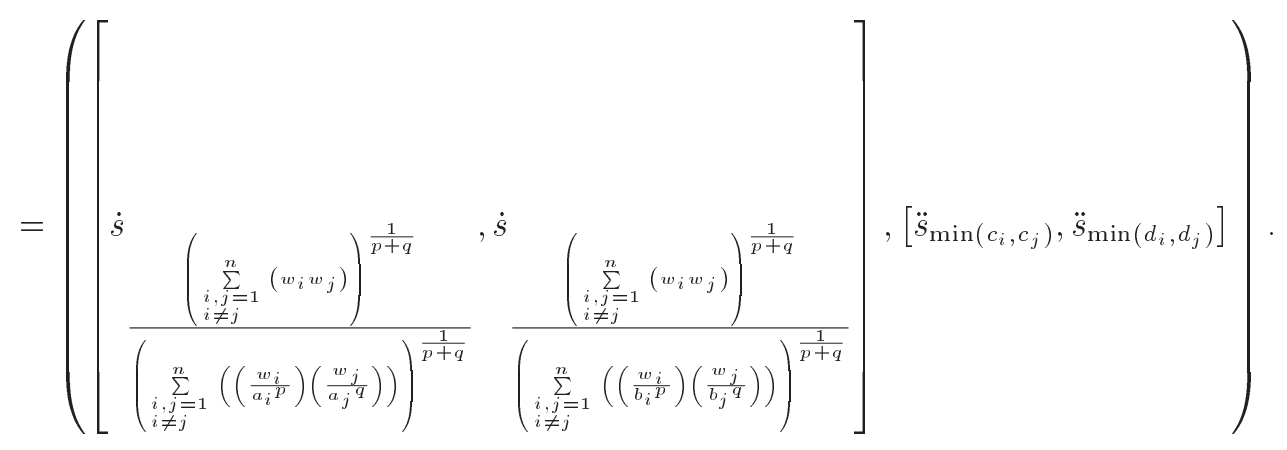

Box XVI

$$
\begin{gathered}
\leq\left(\sum_{\substack{i, j=1 \\
i \neq j}}^{n}\left(\left(\frac{w_{i}}{a_{i}{ }^{p}}\right)\left(\frac{w_{j}}{a_{j^{\prime}}}\right)\right)\right)^{\frac{1}{p+q}} \cdot \text { Then: } \\
\frac{\left(\sum_{\substack{i, j=1 \\
i \neq j}}^{n}\left(w_{i} w_{j}\right)\right)^{\frac{1}{p+q}}}{\left(\sum_{\substack{i, j=1 \\
i \neq j}}^{n}\left(\left(\frac{w_{i}}{a_{i}{ }^{p}}\right)\left(\frac{w_{j}}{a_{j} q}\right)\right)\right)^{\frac{1}{p+q}}}
\end{gathered}
$$$$
\geq \frac{\left(\sum_{\substack{i, j=1 \\ i \neq j}}^{n}\left(w_{i} w_{j}\right)\right)^{\frac{1}{p+q}}}{\left(\sum_{\substack{i, j=1 \\ i \neq j}}^{n}\left(\left(\frac{w_{i}}{a_{i}{ }^{\prime p}}\right)\left(\frac{w_{j}}{a_{j}^{\prime} q}\right)\right)\right)^{\frac{1}{p+q}}} .
$$

Similarly, we also have: 


$$
\begin{gathered}
\frac{\left(\sum_{\substack{i, j=1 \\
i \neq j}}^{n}\left(w_{i} w_{j}\right)\right)^{\frac{1}{p+q}}}{\left(\sum_{\substack{i, j=1 \\
i \neq j}}^{n}\left(\left(\frac{w_{i}}{b_{i} p}\right)\left(\frac{w_{j}}{b_{j}{ }^{q}}\right)\right)\right)^{\frac{1}{p+q}}} \\
\geq \frac{\left(\sum_{\substack{i, j=1 \\
i \neq j}}^{n}\left(w_{i} w_{j}\right)\right)^{\frac{1}{p+q}}}{\left(\sum_{\substack{i, j=1 \\
i \neq j}}^{n}\left(\left(\frac{w_{i}}{b_{i}{ }^{\prime p}}\right)\left(\frac{w_{j}}{b_{j} j^{\prime}}\right)\right)\right)^{\frac{1}{p+q}}} .
\end{gathered}
$$

Further, we have:

$\min \left(\ddot{s}_{c_{1}}, \ddot{s}_{c_{2}}, \ldots, \ddot{s}_{c_{n}}\right) \geq \min \left(\ddot{s}_{c_{1}}^{\prime}, \ddot{s}_{c_{2}}^{\prime}, \ldots, \ddot{s}_{c_{n}}^{\prime}\right)$,

$\min \left(\ddot{s}_{d_{1}}, \ddot{s}_{d_{2}}, \ldots, \ddot{s}_{d_{n}}\right) \geq \min \left(\ddot{s}_{d_{1}}^{\prime}, \ddot{s}_{d_{2}}^{\prime}, \ldots, \ddot{s}_{d_{n}}^{\prime}\right)$.

Finally, we obtain the relation shown in Box XVII.

That is, $\operatorname{DDULIWBHM}^{p, q}\left(\hat{s}_{1}, \hat{s}_{2}, \ldots ., \hat{s}_{n}\right) \quad \geq$ 2DULIWBHM ${ }^{p, q}\left(\hat{s}_{1}^{\prime}, \hat{s}_{2}^{\prime}, \ldots ., \hat{s}_{n}^{\prime}\right)$.

Thus, the proof of Theorem 6 is completed.

Theorem 7 (boundedness). Let:

$$
\hat{s}_{j}=\left(\left[\dot{s}_{a_{j}}, \dot{s}_{b_{j}}\right],\left[\ddot{s}_{c_{j}}, \ddot{s}_{d_{j}}\right]\right) \quad(j=1,2, \ldots, n)
$$

be a collection of 2DULVs, and:

$$
\begin{aligned}
& \hat{s}^{-}=\left(\left[\dot{s}_{\min _{j}\left(a_{j}\right)}, \dot{s}_{\min _{j}\left(b_{j}\right)}\right],\left[\ddot{s}_{\min _{j}\left(c_{j}\right)}, \ddot{s}_{\min _{j}\left(d_{j}\right)}\right]\right) \\
& \hat{s}^{+}=\left(\left[\dot{s}_{\max _{j}\left(a_{j}\right)}, \dot{s}_{\max _{j}\left(b_{j}\right)}\right],\left[\ddot{s}_{\min _{j}\left(c_{j}\right)}, \ddot{s}_{\min _{j}\left(d_{j}\right)}\right]\right) .
\end{aligned}
$$

Then:

$\hat{s}^{-} \leq 2 \operatorname{DULIWBHM}^{p, q}\left(\hat{s}_{1}, \hat{s}_{2}, \ldots, \hat{s}_{n}\right) \leq \hat{s}^{+}$.

Proof. Since $\hat{s}_{j} \geq \hat{s}^{-}$, based on Theorems 4 and 6, we have:

$\operatorname{2DULIWBHM}^{p, q}\left(\hat{s}_{1}, \hat{s}_{2}, \ldots, \hat{s}_{n}\right)$

$$
\geq 2 \operatorname{DULIWBHM}^{p, q}\left(\hat{s}^{-}, \hat{s}^{-}, \ldots, \hat{s}^{-}\right)=\hat{s}^{-} .
$$

Similarly, we also have:

2DULIWBHM ${ }^{p, q}\left(\hat{s}_{1}, \hat{s}_{2}, \ldots, \hat{s}_{n}\right)$

$$
\leq 2 \operatorname{DULIWBHM}^{p, q}\left(\hat{s}^{+}, \hat{s}^{+}, \ldots, \hat{s}^{+}\right)=\hat{s}^{+} .
$$

Then, we obtain:

$\hat{s}^{-} \leq 2 \operatorname{DULIWBHM}^{p, q}\left(\hat{s}_{1}, \hat{s}_{2}, \ldots, \hat{s}_{n}\right) \leq \hat{s}^{+}$,

which completes the proof of Theorem 7 .

In the following, we will study some particular cases of the 2DULIWBHM ${ }^{p, q}$ operator with regard to parameters $p$ and $q$.

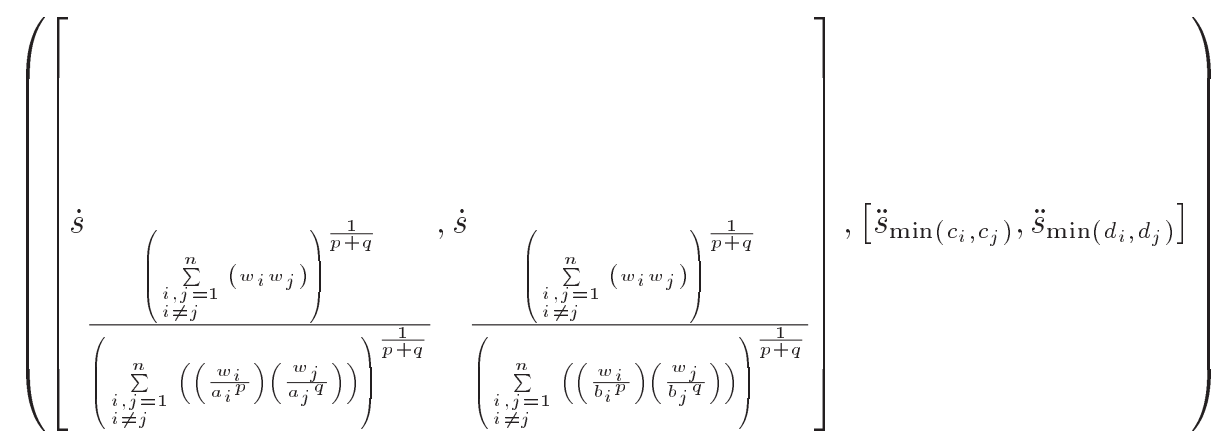

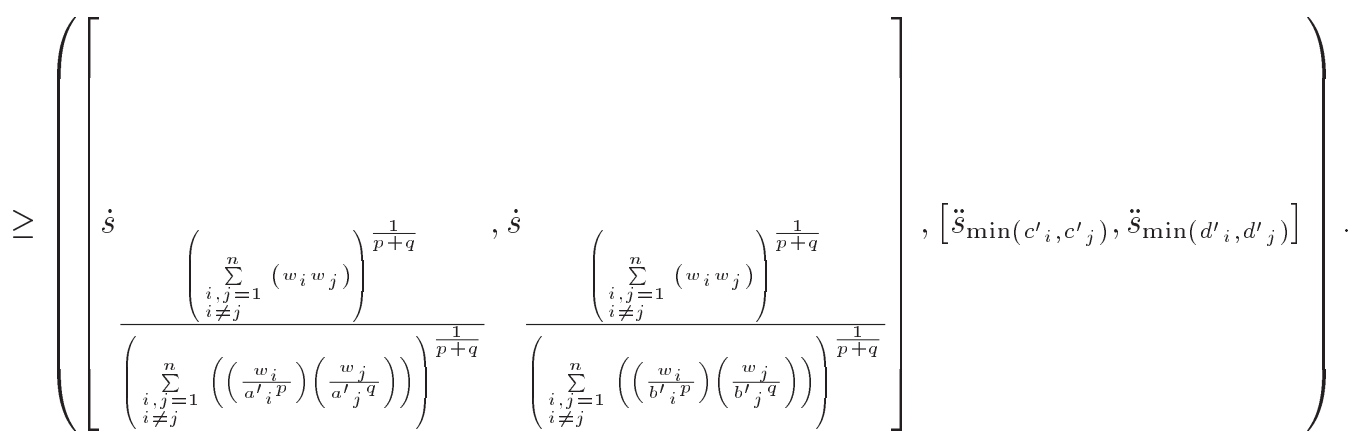




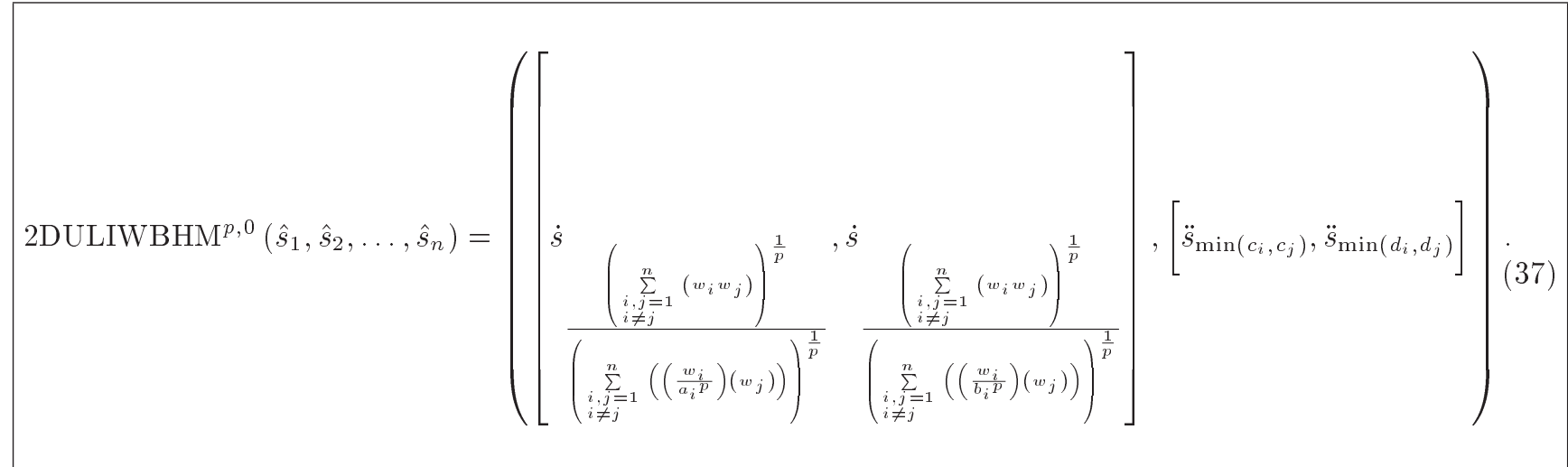

Box XVIII

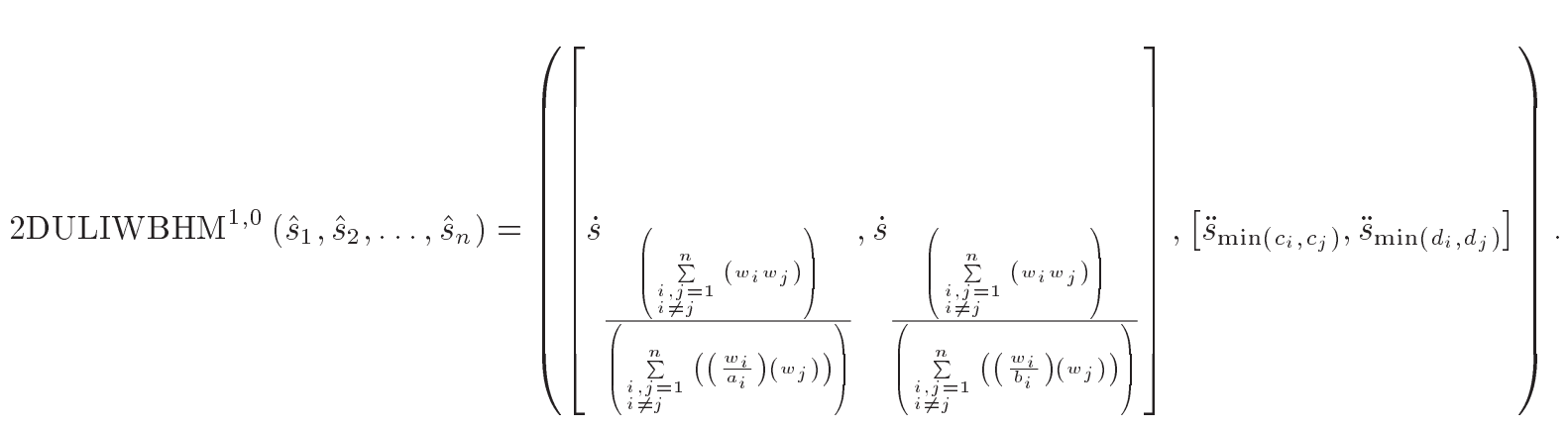

Box XIX

(i) When $q \rightarrow 0$, we can obtain Eq. (37) shown in Box XVIII;

(ii) When $p=1, q \rightarrow 0$, we can obtain Eq. (38) shown in Box XIX;

(iii) When $p \rightarrow 0$, we can obtain Eq. (39) shown in Box XX;

(iv) When $p=q=1$, we can obtain Eq. (40) shown in Box XXI;

\section{A group decision-making approach based on the 2DULIWBHM operator}

In this section, we will apply the 2DULIWBHM operator to solve the MAGDM problems.

A MAGDM problem with $2 \mathrm{DULV}$ is described as follows. Suppose that $\left\{A_{1}, A_{2}, \cdots, A_{m}\right\}$ is a set of alternatives, and $\left\{C_{1}, C_{2}, \cdots, C_{n}\right\}$ is a set of attributes whose weight vector is $w=\left(w_{1}, w_{2}, \cdots, w_{n}\right)^{T}$ with $w_{j} \geq 0, j=1,2, \cdots, n$, and $\sum_{j=1}^{n} w_{j}=1$. Further,

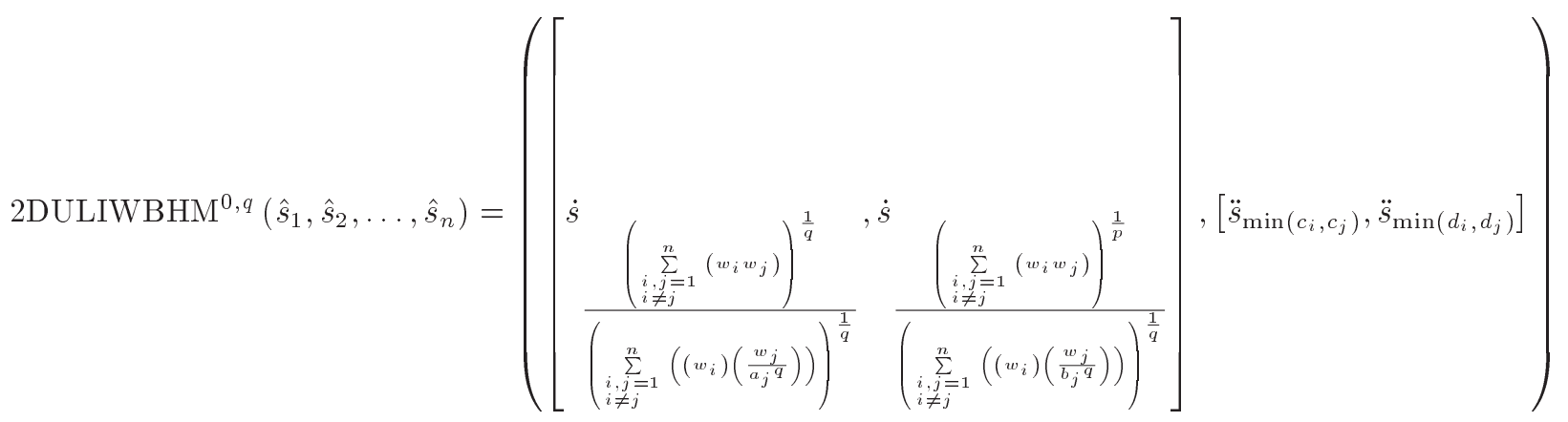




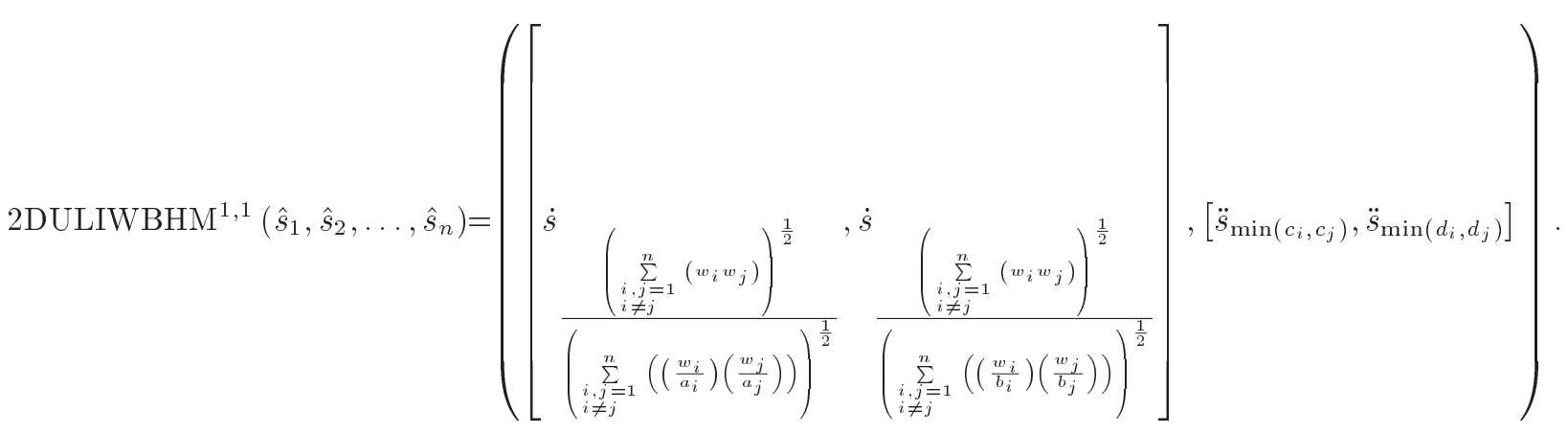

Box XXI

suppose that $D=\left\{D_{1}, D_{2}, \cdots, D_{\mu}\right\}$ is a set of decisionmakers and $\omega=\left(\omega_{1}, \omega_{2}, \cdots, \omega_{\mu}\right)$ is the weight vector of them with $\omega_{k} \geq 0(k=1,2, \cdots, \mu)$, and $\sum_{k=1}^{\mu} \omega_{k}=1$. Let $\hat{S}^{k}=\left[\hat{s}_{i j}^{k}\right]_{m \times n}(k=1,2, \ldots, \mu)$ be the decision matrix of MAGDM problems, where:

$$
\hat{s}_{i j}^{k}=\left(\left[\dot{s}_{a_{i j}^{k}}, \dot{s}_{b_{i j}^{k}}\right]\left[\ddot{s}_{c_{i j}^{k}}, \ddot{s}_{d_{i j}^{k}}\right]\right)
$$

is the evaluation information expressed by the 2DULVs with respect to alternative $A_{i}$ for attribute $C_{j}$ given by the decision maker $D_{k}, \dot{s}_{a_{i j}^{k}}, \dot{s}_{b_{i j}^{k}} \in S_{I}$, $S_{I}=\left(\dot{s}_{0}, \dot{s}_{1}, \ldots, s_{t-1}\right), \dot{s}_{c_{i j}^{k}}, \dot{s}_{d_{i j}^{k}} \in S_{I I}$, and $S_{I I}=$ $\left(\dot{s}_{0}, \dot{s}_{1}, \ldots, s_{L-1}\right)$. Then, the goal of this decision problem is to rank alternatives.

Based on the 2DULIWBHM operator proposed in Section 3, we will give its application in the MAGDM problems and establish the detailed decision-making process, as shown in the following:

Step 1. Normalize the attribute values. In real decision making, the attribute values have two types, i.e., cost attribute and benefit attribute. In order to eliminate the differences between types, we need to convert them to the same type. In general, we need convert cost type to benefit type. If the attribute value $\hat{s}_{i j}^{k}(k=1,2, \ldots, \mu)$ is cost type, it can be transformed to benefit one as shown below (suppose that the transformed attribute value is still expressed by: $\left.\hat{s}_{i j}^{k}=\left(\left[\dot{s}_{a_{i j}^{k}}, \dot{s}_{b_{i j}^{k}}\right]\left[\ddot{s}_{c_{i j}^{k}}, \ddot{s}_{d_{i j}^{k}}\right]\right)\right)$ :

$$
\begin{aligned}
& \hat{s}_{i j}^{k}=\left(\left[\dot{s}_{a_{i j}^{k}}, \dot{s}_{b_{i j}^{k}}\right]\left[\ddot{s}_{c_{i j}^{k}}, \ddot{s}_{d_{i j}^{k}}\right]\right) \\
& =\left\{\begin{array}{c}
\left(\left[\dot{s}_{a_{i j}^{k}}, \dot{s}_{b_{i j}^{k}}\right]\left[\ddot{s}_{c_{i j}^{k}}, \ddot{s}_{d_{i j}^{k}}\right]\right) \\
\text { for benefit attribute } C_{j} \\
\left(\left[\dot{s}_{t-1-b_{i j}^{k}}, \dot{s}_{t-1-a_{i j}^{k}}\right]\left[\ddot{s}_{c_{i j}^{k}}, \ddot{s}_{d_{i j}^{k}}^{k}\right]\right) \\
\text { for cost attribute } C_{j}
\end{array}\right.
\end{aligned}
$$

Step 2. Aggregate the evaluation information of an individual decision-maker with collective information by 2DULIWBHM operator as shown below:

$$
\begin{aligned}
\hat{s}_{i j}= & 2 \operatorname{DULIWBHM}\left(\hat{s}_{i j}^{1}, \hat{s}_{i j}^{2}, \ldots, \hat{s}_{i j}^{\mu}\right) \\
& =\left(\left[\dot{s}_{a_{i j}}, \dot{s}_{b_{i j}}\right]\left[\ddot{s}_{c_{i j}}, \ddot{s}_{d_{i j}}\right]\right) .
\end{aligned}
$$

Step 3. Aggregate the evaluation information of each attribute with the comprehensive evaluation value of each alternative by 2DULIWBHM operator as follows:

$$
\begin{aligned}
\hat{s}_{i} & =2 \operatorname{DULIWBHM}\left(\hat{s}_{i 1}, \hat{s}_{i 2}, \ldots, \hat{s}_{i n}\right) \\
& =\left(\left[\dot{s}_{a_{i}}, \dot{s}_{b_{i}}\right]\left[\ddot{s}_{c_{i}}, \ddot{s}_{d_{i}}\right]\right) .
\end{aligned}
$$

Step 4. Calculate the expectations $E\left(\hat{s}_{i}\right)(i=$ $1,2, \cdots, m)$ of the collective overall values $\hat{s}_{i}(i=$ $1,2, \cdots, m)$ by Eq. (17).

Step 5. Rank all the alternatives $\left\{A_{1}, A_{2}, \cdots, A_{m}\right\}$.

Step 6. End.

\section{An illustrative example}

Example 4. In order to show the application of the proposed method, an example about the land utilization ratio evaluation of four cities $\left\{A_{1}, A_{2}, A_{3}, A_{4}\right\}$ is given. There are three decision-makers, $D_{k}=$ $\{k=1,2,3\}$, who are invited to evaluate the land utilization ratio of cities according to four attributes shown as follows: The proportion of building covering $C_{1}$, the ratio of vegetation covering $C_{2}$, the proportion of traffic covering $C_{3}$, and the ratio of the land $C_{4}$ that should be developed. Suppose that weight vector of three decision-makers is $\omega=(0.4,0.3,0.3)^{T}$, and the attribute weight vector is $w=(0.25,0.25,0.25,0.25)^{T}$. Decision maker $D_{K}$ gives the evaluation value, $\hat{s}_{i j}^{k}$, of city $A_{i}$ with respect to attribute $C_{j}$ by uncertain linguistic variable $\left[\dot{s}_{a_{i j}^{k}}, \dot{s}_{b_{i j}^{k}}\right]$, and then he/she must also evaluate the credibility of the result $\left[\dot{s}_{a_{i j}^{k}}, \dot{s}_{b_{i j}^{k}}\right]$, 
which is also expressed by uncertain linguistic variable $\left[\ddot{s}_{c_{i j}^{k}}, \ddot{s}_{d_{i j}^{k}}\right]$ in another dimension. Then, we can achieve the final evaluation result of city $A_{i}$ with respect to attribute $C_{j}$ by decision-maker $D_{k}$ by the 2DULV $\left(\left[\dot{s}_{a_{i j}^{k}}, \dot{s}_{b_{i j}^{k}}\right]\left[\ddot{s}_{c_{i j}^{k}}, \ddot{s}_{d_{i j}^{k}}\right]\right.$ ) (suppose that Class I linguistic set is $S_{I}=\left(\dot{s}_{0}, \dot{s}_{1}, \dot{s}_{2}, \dot{s}_{3}, \dot{s}_{4}, \dot{s}_{5}, \dot{s}_{6}\right)$, and Class II linguistic set is $S_{I I}=\left(\dot{s}_{0}, \dot{s}_{1}, \dot{s}_{2}, \dot{s}_{3}, \dot{s}_{4}\right)$. For example, decision-maker $D_{1}$ gives the evaluation value $\left[\dot{s}_{5}, \dot{s}_{5}\right]$ for index $C_{1}$ of city $A_{1}$; then, he/she gives the reliability $\left[\ddot{s}_{2}, \ddot{s}_{3}\right]$ of the evaluation value $\left[\dot{s}_{5}, \dot{s}_{5}\right]$; then, the $2 \mathrm{DULV}$ $\left(\left[\dot{s}_{5}, \dot{s}_{5}\right],\left[\ddot{s}_{2}, \ddot{s}_{3}\right]\right)$ is produced. To evaluate all cities with respect to all indexes by all decision-makers, three decision matrices, $\hat{S}^{k}=\left[\hat{s}_{i j}^{k}\right]_{4 \times 4}(k=1,2,3)$, should be constructed, which are listed in Tables 1, 2, and 3 .

Then, the goal is to rank four cities according to the land utilization ratio.

\subsection{The decision-making steps}

To rank the cities, the steps are given as follows:
Step 1. Normalize the attribute values. All the measured values are of benefit type; thus, they do not need to do the standardization.

Step 2. Aggregate the evaluation information of an individual decision-maker (as shown in Box XXII) with collective information by 2DULIWBHM operator expressed in Eq. (42) (without loss of generality, we suppose that $p=q=1$ ).

Step 3. Aggregate the evaluation information of each attribute with the comprehensive evaluation value of each alternative by 2DULIWBHM operator expressed in Eq. (43) (without loss of generality, we let $p=q=1$ ):

$$
\begin{aligned}
& \hat{s}_{1}=\left(\left[\dot{s}_{3.591}, \dot{s}_{4.266}\right]\left[\ddot{s}_{2}, \ddot{s}_{3}\right]\right), \\
& \hat{s}_{2}=\left(\left[\dot{s}_{2.893}, \dot{s}_{3.623}\right]\left[\ddot{s}_{2}, \ddot{s}_{2}\right]\right), \\
& \hat{s}_{3}=\left(\left[\dot{s}_{2.734}, \dot{s}_{3.378}\right]\left[\ddot{s}_{2}, \ddot{s}_{3}\right]\right),
\end{aligned}
$$

$$
\hat{S}=\left(\begin{array}{c}
\left(\left[\dot{s}_{4.644}, \dot{s}_{4.644}\right]\left[\ddot{s}_{2}, \ddot{s}_{3}\right]\right)\left(\left[\dot{s}_{2.569}, \dot{s}_{3.271}\right]\left[\ddot{s}_{2}, \ddot{s}_{2}\right]\right)\left(\left[\dot{s}_{3.873}, \dot{s}_{4.644}\right]\left[\ddot{s}_{2}, \ddot{s}_{3}\right]\right)\left(\left[\dot{s}_{3.830}, \dot{s}_{4.855}\right]\left[\ddot{s}_{1}, \ddot{s}_{2}\right]\right) \\
\left(\left[\dot{s}_{3.589}, \dot{s}_{4.282}\right]\left[\ddot{s}_{2}, \ddot{s}_{3}\right]\right)\left(\left[\dot{s}_{3.359}, \dot{s}_{4.190}\right]\left[\ddot{s}_{2}, \ddot{s}_{2}\right]\right)\left(\left[\dot{s}_{2.133}, \dot{s}_{2.986}\right]\left[\ddot{s}_{2}, \ddot{s}_{3}\right]\right)\left(\left[\dot{s}_{2.872}, \dot{s}_{3.317}\right]\left[\ddot{s}_{1}, \ddot{s}_{2}\right]\right) \\
\left(\left[\dot{s}_{2.569}, \dot{s}_{3.589}\right]\left[\ddot{s}_{2}, \ddot{s}_{3}\right]\right)\left(\left[\dot{s}_{3.830}, \dot{s}_{4.282}\right]\left[\ddot{s}_{2}, \ddot{s}_{2}\right]\right)\left(\left[\dot{s}_{1.773}, \dot{s}_{2.171}\right]\left[\ddot{s}_{2}, \ddot{s}_{3}\right]\right)\left(\left[\dot{s}_{3.633}, \dot{s}_{4.327}\right]\left[\ddot{s}_{1}, \ddot{s}_{2}\right]\right) \\
\left(\left[\dot{s}_{3.576}, \dot{s}_{4.450}\right]\left[\ddot{s}_{2}, \ddot{s}_{3}\right]\right)\left(\left[\dot{s}_{1.805}, \dot{s}_{2.947}\right]\left[\ddot{s}_{2}, \ddot{s}_{2}\right]\right)\left(\left[\dot{s}_{1.840}, \dot{s}_{2.987}\right]\left[\ddot{s}_{2}, \ddot{s}_{3}\right]\right)\left(\left[\dot{s}_{3.589}, \dot{s}_{4.282}\right]\left[\ddot{s}_{1}, \ddot{s}_{2}\right]\right)
\end{array}\right) .
$$

Box XXII

Table 1. Decision matrix $\hat{S}^{1}$.

\begin{tabular}{ccccc}
\hline & $\boldsymbol{C}_{\mathbf{1}}$ & $\boldsymbol{C}_{\mathbf{2}}$ & $\boldsymbol{C}_{\mathbf{3}}$ & $\boldsymbol{C}_{\mathbf{4}}$ \\
\hline$A_{1}$ & $\left(\left[\dot{s}_{5}, \dot{s}_{5}\right],\left[\ddot{s}_{2}, \ddot{s}_{3}\right]\right)$ & $\left(\left[\dot{s}_{2}, \dot{s}_{3}\right],\left[\ddot{s}_{3}, \ddot{s}_{3}\right]\right)$ & $\left(\left[\dot{s}_{4}, \dot{s}_{5}\right],\left[\ddot{s}_{4}, \ddot{s}_{4}\right]\right)$ & $\left(\left[\dot{s}_{3}, \dot{s}_{4}\right],\left[\ddot{s}_{2}, \ddot{s}_{2}\right]\right)$ \\
$A_{2}$ & $\left(\left[\dot{s}_{3}, \dot{s}_{4}\right],\left[\ddot{s}_{2}, \ddot{s}_{3}\right]\right)$ & $\left(\left[\dot{s}_{5}, \dot{s}_{5}\right],\left[\ddot{s}_{3}, \ddot{s}_{3}\right]\right)$ & $\left(\left[\dot{s}_{3}, \dot{s}_{3}\right],\left[\ddot{s}_{4}, \ddot{s}_{4}\right]\right)$ & $\left(\left[\dot{s}_{4}, \dot{s}_{4}\right],\left[\ddot{s}_{2}, \ddot{s}_{2}\right]\right)$ \\
$A_{3}$ & $\left(\left[\dot{s}_{2}, \dot{s}_{3}\right],\left[\ddot{s}_{2}, \ddot{s}_{3}\right]\right)$ & $\left(\left[\dot{s}_{3}, \dot{s}_{4}\right],\left[\ddot{s}_{3}, \ddot{s}_{3}\right]\right)$ & $\left(\left[\dot{s}_{3}, \dot{s}_{4}\right],\left[\ddot{s}_{4}, \ddot{s}_{4}\right]\right)$ & $\left(\left[\dot{s}_{4}, \dot{s}_{5}\right],\left[\ddot{s}_{2}, \ddot{s}_{2}\right]\right)$ \\
$A_{4}$ & $\left(\left[\dot{s}_{5}, \dot{s}_{6}\right],\left[\ddot{s}_{2}, \ddot{s}_{3}\right]\right)$ & $\left(\left[\dot{s}_{1}, \dot{s}_{2}\right],\left[\ddot{s}_{3}, \ddot{s}_{3}\right]\right)$ & $\left(\left[\dot{s}_{2}, \dot{s}_{3}\right],\left[\ddot{s}_{4}, \ddot{s}_{4}\right]\right)$ & $\left(\left[\dot{s}_{3}, \dot{s}_{4}\right],\left[\ddot{s}_{2}, \ddot{s}_{2}\right]\right)$ \\
\hline
\end{tabular}

Table 2. Decision matrix $\hat{S}^{2}$.

\begin{tabular}{ccccc}
\hline & $\boldsymbol{C}_{\mathbf{1}}$ & $\boldsymbol{C}_{\mathbf{2}}$ & $\boldsymbol{C}_{\mathbf{3}}$ & $\boldsymbol{C}_{\mathbf{4}}$ \\
\hline$A_{1}$ & $\left(\left[\dot{s}_{4}, \dot{s}_{4}\right],\left[\ddot{s}_{3}, \ddot{s}_{4}\right]\right)$ & $\left(\left[\dot{s}_{3}, \dot{s}_{4}\right],\left[\ddot{s}_{2}, \ddot{s}_{3}\right]\right)$ & $\left(\left[\dot{s}_{3}, \dot{s}_{4}\right],\left[\ddot{s}_{2}, \ddot{s}_{3}\right]\right)$ & $\left(\left[\dot{s}_{5}, \dot{s}_{6}\right],\left[\ddot{s}_{3}, \ddot{s}_{4}\right]\right)$ \\
$A_{2}$ & $\left(\left[\dot{s}_{4}, \dot{s}_{5}\right],\left[\ddot{s}_{3}, \ddot{s}_{4}\right]\right)$ & $\left(\left[\dot{s}_{2}, \dot{s}_{3}\right],\left[\ddot{s}_{2}, \ddot{s}_{3}\right]\right)$ & $\left(\left[\dot{s}_{4}, \dot{s}_{5}\right],\left[\ddot{s}_{2}, \ddot{s}_{3}\right]\right)$ & $\left(\left[\dot{s}_{2}, \dot{s}_{3}\right],\left[\ddot{s}_{3}, \ddot{s}_{4}\right]\right)$ \\
$A_{3}$ & $\left(\left[\dot{s}_{3}, \dot{s}_{4}\right],\left[\ddot{s}_{3}, \ddot{s}_{4}\right]\right)$ & $\left(\left[\dot{s}_{4}, \dot{s}_{4}\right],\left[\ddot{s}_{2}, \ddot{s}_{3}\right]\right)$ & $\left(\left[\dot{s}_{2}, \dot{s}_{3}\right],\left[\ddot{s}_{2}, \ddot{s}_{3}\right]\right)$ & $\left(\left[\dot{s}_{3}, \dot{s}_{4}\right],\left[\ddot{s}_{3}, \ddot{s}_{4}\right]\right)$ \\
$A_{4}$ & $\left(\left[\dot{s}_{5}, \dot{s}_{5}\right],\left[\ddot{s}_{3}, \ddot{s}_{4}\right]\right)$ & $\left(\left[\dot{s}_{4}, \dot{s}_{5}\right],\left[\ddot{s}_{2}, \ddot{s}_{3}\right]\right)$ & $\left(\left[\dot{s}_{1}, \dot{s}_{2}\right],\left[\ddot{s}_{2}, \ddot{s}_{3}\right]\right)$ & $\left(\left[\dot{s}_{4}, \dot{s}_{4}\right],\left[\ddot{s}_{3}, \ddot{s}_{4}\right]\right)$ \\
\hline
\end{tabular}

Table 3. Decision matrix $\hat{S}^{3}$.

\begin{tabular}{ccccc}
\hline & $\boldsymbol{C}_{\mathbf{1}}$ & $\boldsymbol{C}_{\mathbf{2}}$ & $\boldsymbol{C}_{\mathbf{3}}$ & $\boldsymbol{C}_{\mathbf{4}}$ \\
\hline$A_{1}$ & $\left(\left[\dot{s}_{5}, \dot{s}_{5}\right],\left[\ddot{s}_{2}, \ddot{s}_{3}\right]\right)$ & $\left(\left[\dot{s}_{3}, \dot{s}_{3}\right],\left[\ddot{s}_{2}, \ddot{s}_{2}\right]\right)$ & $\left(\left[\dot{s}_{4}, \dot{s}_{4}\right],\left[\ddot{s}_{3}, \ddot{s}_{4}\right]\right)$ & $\left(\left[\dot{s}_{4}, \dot{s}_{5}\right],\left[\ddot{s}_{1}, \ddot{s}_{2}\right]\right)$ \\
$A_{2}$ & $\left(\left[\dot{s}_{4}, \dot{s}_{4}\right],\left[\ddot{s}_{2}, \ddot{s}_{3}\right]\right)$ & $\left(\left[\dot{s}_{4}, \dot{s}_{5}\right],\left[\ddot{s}_{2}, \ddot{s}_{2}\right]\right)$ & $\left(\left[\dot{s}_{1}, \dot{s}_{2}\right],\left[\ddot{s}_{3}, \ddot{s}_{4}\right]\right)$ & $\left(\left[\dot{s}_{3}, \dot{s}_{3}\right],\left[\ddot{s}_{1}, \ddot{s}_{2}\right]\right)$ \\
$A_{3}$ & $\left(\left[\dot{s}_{3}, \dot{s}_{4}\right],\left[\ddot{s}_{2}, \ddot{s}_{3}\right]\right)$ & $\left(\left[\dot{s}_{5}, \dot{s}_{5}\right],\left[\ddot{s}_{2}, \ddot{s}_{2}\right]\right)$ & $\left(\left[\dot{s}_{1}, \dot{s}_{1}\right],\left[\ddot{s}_{3}, \ddot{s}_{4}\right]\right)$ & $\left(\left[\dot{s}_{4}, \dot{s}_{4}\right],\left[\ddot{s}_{1}, \ddot{s}_{2}\right]\right)$ \\
$A_{4}$ & $\left(\left[\dot{s}_{2}, \dot{s}_{3}\right],\left[\ddot{s}_{2}, \ddot{s}_{3}\right]\right)$ & $\left(\left[\dot{s}_{2}, \dot{s}_{3}\right],\left[\ddot{s}_{2}, \ddot{s}_{2}\right]\right)$ & $\left(\left[\dot{s}_{4}, \dot{s}_{5}\right],\left[\ddot{s}_{3}, \ddot{s}_{4}\right]\right)$ & $\left(\left[\dot{s}_{4}, \dot{s}_{5}\right],\left[\ddot{s}_{1}, \ddot{s}_{2}\right]\right)$ \\
\hline
\end{tabular}


$\hat{s}_{4}=\left(\left[\dot{s}_{2.460}, \dot{s}_{3.554}\right]\left[\ddot{s}_{1}, \ddot{s}_{2}\right]\right)$.

Step 4. Calculate the expectations $E\left(\hat{s}_{i}\right)(i=$ $1,2,3,4)$ of the collective overall values $\hat{s}_{i}(i=$ $1,2,3,4)$ :

$E\left(\hat{s}_{1}\right)=0.409, \quad E\left(\hat{s}_{2}\right)=0.271$,

$E\left(\hat{s}_{3}\right)=0.318, \quad E\left(\hat{s}_{4}\right)=0.188$.

Step 5. Rank the alternatives. Based on Definition 5 , we can rank the alternatives $\left\{A_{1}, A_{2}, A_{3}, A_{4}\right\}$ shown as follows:

$A_{1} \succ A_{3} \succ A_{2} \succ A_{4}$.

Therefore, the best alternative is $A_{1}$.

Step 6. End.

\subsection{The influence of the parameters $p$ and $q$ on decision making result of this example}

In order to demonstrate the influence of parameters $p$ and $q$ on decision-making in this example, different values of $p$ and $q$ in Steps 2 and 3 are used to rank the alternatives. The ranking results are shown in Table 4.

The ranking results with different parameters $p$ and $q$ are slightly different; however, the best choice is always $A_{1}$ and the worst is $A_{4}$, indicating that the 2DULIWBHM operator can reflect the decisionmaker's subjective preferences. When $p=q$, we can find that the ranking of the alternatives is all the same. In addition, because the score functions of alternatives become smaller and smaller as the parameter $p$ and $q$ increase, we can regard parameters $p$ and $q$ as decision-makers' risk attitude. When decision-

Table 4. Ordering of the alternatives by utilizing different $p$ and $q$.

\begin{tabular}{|c|c|c|}
\hline Parameters & Expectations $E\left(\hat{s}_{i}\right)$ & Ranking \\
\hline$p=q=\frac{1}{2}$ & $\begin{aligned} E\left(\hat{s}_{1}\right) & =0.413, E\left(\hat{s}_{2}\right)=0.276 \\
E\left(\hat{s}_{3}\right) & =0.326, E\left(\hat{s}_{4}\right)=0.193\end{aligned}$ & $A_{1} \succ A_{3} \succ A_{2} \succ A_{4}$ \\
\hline$p=q=1$ & $\begin{aligned} E\left(\hat{s}_{1}\right) & =0.409, E\left(\hat{s}_{2}\right)=0.271 \\
E\left(\hat{s}_{3}\right) & =0.318, E\left(\hat{s}_{4}\right)=0.188\end{aligned}$ & $A_{1} \succ A_{3} \succ A_{2} \succ A_{4}$ \\
\hline$p=2, q=1$ & $\begin{aligned} E\left(\hat{s}_{1}\right) & =0.402, E\left(\hat{s}_{2}\right)=0.262 \\
E\left(\hat{s}_{3}\right) & =0.301, E\left(\hat{s}_{4}\right)=0.178\end{aligned}$ & $A_{1} \succ A_{3} \succ A_{2} \succ A_{4}$ \\
\hline$p=1, q=2$ & $\begin{aligned} E\left(\hat{s}_{1}\right) & =0.402, E\left(\hat{s}_{2}\right)=0.262 \\
E\left(\hat{s}_{3}\right) & =0.301, E\left(\hat{s}_{4}\right)=0.178\end{aligned}$ & $A_{1} \succ A_{3} \succ A_{2} \succ A_{4}$ \\
\hline$p=2, q=2$ & $\begin{aligned} E\left(\hat{s}_{1}\right) & =0.402, E\left(\hat{s}_{2}\right)=0.263 \\
E\left(\hat{s}_{3}\right) & =0.304, E\left(\hat{s}_{4}\right)=0.178\end{aligned}$ & $A_{1} \succ A_{3} \succ A_{2} \succ A_{4}$ \\
\hline$p=1, q=5$ & $\begin{aligned} E\left(\hat{s}_{1}\right) & =0.371, E\left(\hat{s}_{2}\right)=0.218 \\
E\left(\hat{s}_{3}\right) & =0.217, E\left(\hat{s}_{4}\right)=0.145\end{aligned}$ & $A_{1} \succ A_{2} \succ A_{3} \succ A_{4}$ \\
\hline$p=5, q=1$ & $\begin{aligned} E\left(\hat{s}_{1}\right) & =0.371, E\left(\hat{s}_{2}\right)=0.218 \\
E\left(\hat{s}_{3}\right) & =0.217, E\left(\hat{s}_{4}\right)=0.145\end{aligned}$ & $A_{1} \succ A_{2} \succ A_{3} \succ A_{4}$ \\
\hline$p=5, q=5$ & $\begin{aligned} E\left(\hat{s}_{1}\right) & =0.386, E\left(\hat{s}_{2}\right)=0.244 \\
E\left(\hat{s}_{3}\right) & =0.274, E\left(\hat{s}_{4}\right)=0.155\end{aligned}$ & $A_{1} \succ A_{3} \succ A_{2} \succ A_{4}$ \\
\hline$p=1, q=10$ & $\begin{array}{l}E\left(\hat{s}_{1}\right)=0.329, E\left(\hat{s}_{2}\right)=0.177 \\
E\left(\hat{s}_{3}\right)=0.159, E\left(\hat{s}_{4}\right)=0.121\end{array}$ & $A_{1} \succ A_{2} \succ A_{3} \succ A_{4}$ \\
\hline$p=10, q=1$ & $\begin{array}{c}E\left(\hat{s}_{1}\right)=0.329, E\left(\hat{s}_{2}\right)=0.177 \\
E\left(\hat{s}_{3}\right)=0.159, E\left(\hat{s}_{4}\right)=0.121\end{array}$ & $A_{1} \succ A_{2} \succ A_{3} \succ A_{4}$ \\
\hline$p=10, q=10$ & $\begin{array}{l}E\left(\hat{s}_{1}\right)=0.369, E\left(\hat{s}_{2}\right)=0.227 \\
E\left(\hat{s}_{3}\right)=0.253, E\left(\hat{s}_{4}\right)=0.139\end{array}$ & $A_{1} \succ A_{3} \succ A_{2} \succ A_{4}$ \\
\hline
\end{tabular}


makers are optimistic, the small values of parameters $p$ and $q$ can be adopted, and when decision-makers are pessimistic, the big values of parameters $p$ and $q$ can be used. Therefore, in real practical decision-making situations, decision-makers can choose an appropriate value in accordance with their risk preferences. When $p \geq 5$ or $q \geq 5$ and $p \neq q$, the ranking changes to $A_{1} \succ A_{2} \succ A_{3} \succ A_{4}$.

\subsection{The verification of the validity}

To prove the effectiveness of the improved method in this paper, the same illustrative example is solved using the two existing MAGDM methods: the 2-Dimensional Uncertain Linguistic Weighted Geometric Heronian Mean (2DULWGHM) operator proposed by Liu and Chu [46]; 2-Dimensional Uncertain Linguistic Power Generalized Weighted Aggregation (2DULPGWA) operator proposed by Liu and $\mathrm{Yu}[20]$.

For convenience, we let $p=q=1$; then, the final ranking orders of the alternatives obtained by these methods are described in Table 5 .

From Table 5, we can find that there are the same ranking results using four methods. Therefore, the method in this paper is effective and feasible.

\subsection{Further discussion on the proposed methods}

According to the above subsections, the validity of our proposed method has been confirmed. However, it has been found that they have the same ranking results; hence, it is difficult to illustrate the advantages of our method and the drawbacks of the existing methods in some situations. Therefore, two examples are given to show the advantages of our proposed method based on the 2DULIWBHM operator. These examples are particular cases of Example 4 given as follows.

Example 5. For easily calculation, the evaluation information of decision-maker $D_{2}$ who is invited to evaluate the land utilization ratio of four cities $\left\{A_{1}, A_{2}, A_{3}, A_{4}\right\}$ is adopted according to the four attributes, i.e., the proportion of building covering $C_{1}$, the ratio of vegetation covering $C_{2}$, the proportion of traffic covering $C_{3}$, and the ratio of land $C_{4}$ that should be developed. Suppose that weight vector of the attribute is $w=(0.25,0.25,0.25,0.25)^{T}$. Decision-maker $D_{2}$ gives the evaluation value $\hat{s}_{i j}$ of city $A_{i}$ with respect to attribute $C_{j}$, which is expressed by the 2-dimensional uncertain linguistic variables (suppose that Class I linguistic set is $S_{I}=$ $\left(\dot{s}_{0}, \dot{s}_{1}, \dot{s}_{2}, \dot{s}_{3}, \dot{s}_{4}, \dot{s}_{5}, \dot{s}_{6}\right)$, and Class II linguistic set is $S_{I I}=\left(\dot{s}_{0}, \dot{s}_{1}, \dot{s}_{2}, \dot{s}_{3}, \dot{s}_{4}\right)$. In addition, decision matrices $\hat{S}=\left[\hat{s}_{i j}\right]_{4 \times 4}$ are listed in Table 6 , where $\hat{s}_{i j}$ can be expressed as $\left(\left[\dot{s}_{a_{i j}}, \dot{s}_{b_{i j}}\right]\left[\ddot{s}_{c_{i j}}, \ddot{s}_{d_{i j}}\right]\right)$. Then, the goal is to rank four cities according to the land utilization ratio.

The ranking results are shown in Table 7 , from which, we can find that when $p=q=1$, the best alternative to the proposed method is $A_{3}$ and other is $A_{1}$. We can explain that the ranking result produced by the proposed method is more reasonable than that produced by Liu and Chu [46], because the proposed method considers the effects of unreasonable data from biased decision-makers, such as too high or too low arguments. In this example, because Class I uncertain linguistic variable of $\hat{s}_{12}$ and $\hat{s}_{14}$ is $\left[\dot{s}_{5}, \dot{s}_{6}\right]$, which are quite larger than the others. The proposed method can

Table 5. Ranking results by different methods.

\begin{tabular}{cccc}
\hline Method & Aggregation operator & Score values $\boldsymbol{S}\left(\boldsymbol{Z}_{\boldsymbol{i}}\right)$ & Ranking \\
\hline \multirow{2}{*}{ Liu and Chu [46] } & 2DULWGHM & $E\left(\hat{s}_{1}\right)=0.429, E\left(\hat{s}_{2}\right)=0.302$, & $A_{1} \succ A_{3} \succ A_{2} \succ A_{4}$ \\
& $E\left(\hat{s}_{3}\right)=0.361, E\left(\hat{s}_{4}\right)=0.221$ & \\
& & $E\left(\hat{s}_{1}\right)=0.328, E\left(\hat{s}_{2}\right)=0.177$, & $A_{1} \succ A_{3} \succ A_{2} \succ A_{4}$ \\
Liu and Yu [20] & 2DULPGWA & $E\left(\hat{s}_{3}\right)=0.159, E\left(\hat{s}_{4}\right)=0.121$ & \\
& & \\
& & & \\
Proposed method & 2DULIWBHM & $E\left(\hat{s}_{1}\right)=0.427, E\left(\hat{s}_{2}\right)=0.298$, & $A_{1} \succ A_{3} \succ A_{2} \succ A_{4}$ \\
\hline
\end{tabular}

Table 6. Decision matrix $\hat{S}$ of Example 5 .

\begin{tabular}{ccccc}
\hline & $\boldsymbol{C}_{\mathbf{1}}$ & $\boldsymbol{C}_{\mathbf{2}}$ & $\boldsymbol{C}_{\mathbf{3}}$ & $\boldsymbol{C}_{4}$ \\
\hline$A_{1}$ & $\left(\left[\dot{s}_{4}, \dot{s}_{4}\right],\left[\ddot{s}_{2}, \ddot{s}_{3}\right]\right)$ & $\left(\left[\dot{s}_{5}, \dot{s}_{6}\right],\left[\ddot{s}_{2}, \ddot{s}_{2}\right]\right)$ & $\left(\left[\dot{s}_{3}, \dot{s}_{4}\right],\left[\ddot{s}_{3}, \ddot{s}_{4}\right]\right)$ & $\left(\left[\dot{s}_{5}, \dot{s}_{6}\right],\left[\ddot{s}_{1}, \ddot{s}_{2}\right]\right)$ \\
$A_{2}$ & $\left(\left[\dot{s}_{4}, \dot{s}_{5}\right],\left[\ddot{s}_{2}, \ddot{s}_{3}\right]\right)$ & $\left(\left[\dot{s}_{2}, \dot{s}_{3}\right],\left[\ddot{s}_{2}, \ddot{s}_{2}\right]\right)$ & $\left(\left[\dot{s}_{4}, \dot{s}_{5}\right],\left[\ddot{s}_{3}, \ddot{s}_{4}\right]\right)$ & $\left(\left[\dot{s}_{2}, \dot{s}_{3}\right],\left[\ddot{s}_{1}, \ddot{s}_{2}\right]\right)$ \\
$A_{3}$ & $\left(\left[\dot{s}_{3}, \dot{s}_{4}\right],\left[\ddot{s}_{2}, \ddot{s}_{3}\right]\right)$ & $\left(\left[\dot{s}_{3}, \dot{s}_{4}\right],\left[\ddot{s}_{2}, \ddot{s}_{2}\right]\right)$ & $\left(\left[\dot{s}_{2}, \dot{s}_{3}\right],\left[\ddot{s}_{3}, \ddot{s}_{4}\right]\right)$ & $\left(\left[\dot{s}_{3}, \dot{s}_{4}\right],\left[\ddot{s}_{1}, \ddot{s}_{2}\right]\right)$ \\
$A_{4}$ & $\left(\left[\dot{s}_{5}, \dot{s}_{5}\right],\left[\ddot{s}_{2}, \ddot{s}_{3}\right]\right)$ & $\left(\left[\dot{s}_{4}, \dot{s}_{5}\right],\left[\ddot{s}_{2}, \ddot{s}_{2}\right]\right)$ & $\left(\left[\dot{s}_{3}, \dot{s}_{4}\right],\left[\ddot{s}_{3}, \ddot{s}_{4}\right]\right)$ & $\left(\left[\dot{s}_{4}, \dot{s}_{4}\right],\left[\ddot{s}_{1}, \ddot{s}_{2}\right]\right)$ \\
\hline
\end{tabular}


Table 7. Ranking results by different methods for Example 5.

\begin{tabular}{cccc}
\hline Method by & Aggregation operator & Expected values $\boldsymbol{E}\left(\hat{\boldsymbol{s}}_{\boldsymbol{i}}\right)$ & Ranking \\
\hline \multirow{2}{*}{ Liu and Chu [46] } & \multirow{2}{*}{ 2DULWGHM $(p=q=1)$} & $E\left(\hat{s}_{1}\right)=0.4837, E\left(\hat{s}_{2}\right)=0.2941$, & $A_{1} \succ A_{3} \succ A_{2} \succ A_{4}$ \\
& & $E\left(\hat{s}_{3}\right)=0.4748, E\left(\hat{s}_{4}\right)=0.2662$ & \\
& & \\
Proposed method & 2DULIWBHM $(p=q=1)$ & \\
& & $E\left(\hat{s}_{1}\right)=0.4653, E\left(\hat{s}_{3}\right)=0.4657, E\left(\hat{s}_{4}\right)=0.2608$ & $A_{3} \succ A_{1} \succ A_{2} \succ A_{4}$ \\
\hline
\end{tabular}

better process this type of decision problem and relieve the effects of unreasonable data, while the method of Liu and Chu [46] cannot eliminate the effect of extreme cases; thus, the ranking result produced by the proposed method is more reasonable than the result produced by the method of Liu and Chu [46].

Example 6. Similar to Example 5, the evaluation results of decision-maker $D_{3}$ who is invited to evaluate the land utilization ratio of four cities $\left\{A_{1}, A_{2}, A_{3}, A_{4}\right\}$ are adopted according to the four attributes: the proportion of building covering $C_{1}$, the ratio of vegetation covering $C_{2}$, the proportion of traffic covering $C_{3}$, and the ratio of the land $C_{4}$ that should be developed. Suppose that weight vector of the attribute is $w=(0.25,0.25,0.25,0.25)^{T}$. Decision-maker $D_{3}$ gives evaluation value, $\hat{s}_{i j}$, of city $A_{i}$ with respect to attribute $C_{j}$, which is expressed by the 2-dimensional uncertain linguistic variables. Suppose that Class I linguistic set is $S_{I}=\left(\dot{s}_{0}, \dot{s}_{1}, \dot{s}_{2}, \dot{s}_{3}, \dot{s}_{4}, \dot{s}_{5}, \dot{s}_{6}\right)$ and Class II linguistic set is $S_{I I}=\left(\dot{s}_{0}, \dot{s}_{1}, \dot{s}_{2}, \dot{s}_{3}, \dot{s}_{4}\right)$. In addition, decision matrices $\hat{S}=\left[\hat{s}_{i j}\right]_{4 \times 4}$ are listed in Table 8 , where $\hat{s}_{i j}$ can be expressed as $\left(\left[\dot{s}_{a_{i j}}, \dot{s}_{b_{i j}}\right]\left[\ddot{s}_{c_{i j}}, \ddot{s}_{d_{i j}}\right]\right)$. Then, the goal is to rank the four cities according to the land utilization ratio.

The ranking results are shown in Table 9, from which we can find that the best alternative of the proposed method is $A_{1}$ and the other is $A_{3}$. Obviously, these two methods can relieve the effect of unreasonable data, such as too high or too low data given by biased decision-makers; however, the only difference between them is that the proposed method can also consider the relationship of attributes. In real decisionmaking problems, it is common that there is the relationship among attributes. Therefore, the proposed method in this paper is more reasonable than that proposed by Liu et al. [20]. In this example, there is the interrelationship among four attributes; therefore, the ranking result produced by the proposed method is more reasonable than the result produced by the method of Liu and Yu [20].

According to the above analysis, the comparison of the proposed method based on 2DULIWBHM operator with the other methods can be described as follows (see Table 10):

1. Compared with the method in [46] based on the 2DULWGHM operator, we can find that these two methods adopt the same operational rules which are more accurate in operations. At the same time, these two methods all consider the interrelationship for input arguments. However, the method in [46] cannot consider the case when the given arguments

Table 8. Decision matrix $\hat{S}$ of Example 6 .

\begin{tabular}{ccccc}
\hline & $\boldsymbol{C}_{\mathbf{1}}$ & $\boldsymbol{C}_{\mathbf{2}}$ & $\boldsymbol{C}_{\mathbf{3}}$ & $\boldsymbol{C}_{\mathbf{4}}$ \\
\hline$A_{1}$ & $\left(\left[\dot{s}_{4}, \dot{s}_{5}\right],\left[\ddot{s}_{2}, \ddot{s}_{3}\right]\right)$ & $\left(\left[\dot{s}_{3}, \dot{s}_{5}\right],\left[\ddot{s}_{2}, \ddot{s}_{2}\right]\right)$ & $\left(\left[\dot{s}_{5}, \dot{s}_{5}\right],\left[\ddot{s}_{3}, \ddot{s}_{3}\right]\right)$ & $\left(\left[\dot{s}_{4}, \dot{s}_{5}\right],\left[\ddot{s}_{1}, \ddot{s}_{2}\right]\right)$ \\
$A_{2}$ & $\left(\left[\dot{s}_{4}, \dot{s}_{4}\right],\left[\ddot{s}_{2}, \ddot{s}_{3}\right]\right)$ & $\left(\left[\dot{s}_{4}, \dot{s}_{5}\right],\left[\ddot{s}_{2}, \ddot{s}_{2}\right]\right)$ & $\left(\left[\dot{s}_{1}, \dot{s}_{2}\right],\left[\ddot{s}_{3}, \ddot{s}_{3}\right]\right)$ & $\left(\left[\dot{s}_{3}, \dot{s}_{3}\right],\left[\ddot{s}_{1}, \ddot{s}_{2}\right]\right)$ \\
$A_{3}$ & $\left(\left[\dot{s}_{3}, \dot{s}_{4}\right],\left[\ddot{s}_{2}, \ddot{s}_{3}\right]\right)$ & $\left(\left[\dot{s}_{5}, \dot{s}_{5}\right],\left[\ddot{s}_{2}, \ddot{s}_{2}\right]\right)$ & $\left(\left[\dot{s}_{2}, \dot{s}_{3}\right],\left[\ddot{s}_{3}, \ddot{s}_{3}\right]\right)$ & $\left(\left[\dot{s}_{4}, \dot{s}_{4}\right],\left[\ddot{s}_{1}, \ddot{s}_{2}\right]\right)$ \\
$A_{4}$ & $\left(\left[\dot{s}_{2}, \dot{s}_{3}\right],\left[\ddot{s}_{2}, \ddot{s}_{3}\right]\right)$ & $\left(\left[\dot{s}_{2}, \dot{s}_{3}\right],\left[\ddot{s}_{2}, \ddot{s}_{2}\right]\right)$ & $\left(\left[\dot{s}_{4}, \dot{s}_{5}\right],\left[\ddot{s}_{3}, \ddot{s}_{3}\right]\right)$ & $\left(\left[\dot{s}_{4}, \dot{s}_{5}\right],\left[\ddot{s}_{1}, \ddot{s}_{2}\right]\right)$ \\
\hline
\end{tabular}

Table 9. Ranking results by different methods for Example 6 .

\begin{tabular}{cccc}
\hline Method by & Aggregation operator & Expected values $\boldsymbol{E}\left(\hat{\boldsymbol{s}}_{\boldsymbol{i}}\right)$ & Ranking \\
\hline \multirow{2}{*}{ Liu and Yu [20] } & 2DULPGWA & $E\left(\hat{s}_{1}\right)=0.4676, E\left(\hat{s}_{2}\right)=0.2790$, & $A_{3} \succ A_{1} \succ A_{2} \succ A_{4}$ \\
& $E\left(\hat{s}_{3}\right)=0.4678, E\left(\hat{s}_{4}\right)=0.2167$ & \\
& & \\
Proposed method & 2DULIWBHM $(p=q=1)$ & $E\left(\hat{s}_{1}\right)=0.4632, E\left(\hat{s}_{2}\right)=0.2317$, & $A_{1} \succ A_{3} \succ A_{2} \succ A_{4}$ \\
& & $E\left(\hat{s}_{3}\right)=0.4424, E\left(\hat{s}_{4}\right)=0.2033$ & \\
\hline
\end{tabular}


Table 10. The comparisons of different methods.

\begin{tabular}{lcc}
\hline Methods by & $\begin{array}{c}\text { Whether considers } \\
\text { interrelationship of } \\
\text { two attributes }\end{array}$ & $\begin{array}{c}\text { Whether considers } \\
\text { the effect from too high } \\
\text { or too low arguments }\end{array}$ \\
\hline Liu and Chu [46] & Yes & No \\
Liu and Yu [20] & No & Yes \\
2DULIWBHM & Yes & Yes \\
\hline
\end{tabular}

have a great difference; in other words, it cannot eliminate the effects of unreasonable data from biased decision-makers. For instance, if the given data are too high or too low, it cannot achieve the reasonable result. Obviously, the proposed method is more flexible and general to solve the MAGDM problems than the method proposed by Liu and Chu [46].

2. Compared with the method in [20] based on the 2DULPGWA operator, the two methods both can deal with the problems about unreasonable data given by biased decision-makers. However, the attributes are independent in the method proposed in [20], and the interrelationship between them is not considered. In many times, there exists much relevance between the given arguments. The proposed method in this paper can easily solve this problem with different parameters $p$ and $q$ using BM; thus, our method is more flexible and reasonable than the method in [20].

According to the comparisons and analyses above, our method can combine the advantages of the two methods efficiently. In other words, the proposed method based on the 2DULIWBHM operator in this paper is better than the other existing methods for aggregating the 2DULVs. Of course, there is a shortcoming in the proposed method, i.e., it is more complex in the calculation process than the method in [20].

\section{Conclusion}

Because 2DULVs can express fuzzy information better, this study extended the Bonferroni Mean (BM) operator to 2DULVs and proposed the 2-Dimensional Uncertain Linguistic Weighted Bonferroni Mean (2DULWBM). However, it cannot consider the case when the given arguments are too high or too low. Therefore, the 2DULWBM was combined with harmonic mean to solve this problem. Then, some of their desirable characteristics, such as idempotency, monotonicity, boundedness, and commutativity, were discussed. Further, some particular cases of these operators were analyzed, and a method was proposed for the MAGDM based on the 2DULIWBHM operators. Compared with the existing methods, the proposed method is more general than some existing methods. The significant advantages of the proposed method include (1) capturing the interrelationship among the input arguments that have the flexibility with Bonferroni mean parameters $p, q$ and (2) considering the case when the given arguments are too high or too low. For future studies, it is necessary and significant to apply these operators to solve the real decision-making problems such as fuzzy cluster analysis, uncertain programming, etc.

\section{Acknowledgment}

This paper is supported by the National Natural Science Foundation of China (Nos. 71471172 and 71271124), the Special Funds of Taishan Scholars Project of Shandong Province (No. ts201511045), Shandong Provincial Social Science Planning Project (Nos. 16CGLJ31 and 16CKJJ27), the Teaching Reform Research Project of Undergraduate Colleges and Universities in Shandong Province (No. 2015Z057), and Key research and development program of Shandong Province (No. 2016GNC110016).

\section{References}

1. Churchman, C.W., Ackoff, R.L., and Arnoff, E.L., Introduction to Operations Research, Wiley, New York (1957).

2. Chen, T.Y., Chang, C.H., and Lu, J.R. "The extended QUALIFLEX method for multiple criteria decision analysis based on interval type-2 fuzzy sets and applications to medical decision making", Eur J Oper Res, 226(3), pp. 615-625 (2013).

3. Chuu, S.J. "Interactive group decision-making using a fuzzy linguistic approach for evaluating the flexibility in a supply chain", Eur J Oper Res, 213(1), pp. 279289 (2011).

4. Liu, F., Wang, W.G., and Zhang, Z.X. "A goal programming model for incomplete interval multiplicative preference relations and its application in group decision-making", Eur J Oper Res, 218(3), pp. 747754 (2012).

5. Ye, J. "Fuzzy decision-making method based on the weighted correlation coefficient under intuitionistic fuzzy environment", Eur J Oper Res, 205(1), pp. 202204 (2010). 
6. Zhang, C., Zhou, G.Z., and Zhu, W.D. "Research on peer review system for the national science foundation based on two-dimensional semantics evidence reasoning", China Soft Sci, 2, pp. 176-182 (2011).

7. Liu, P.D. and Tang, G.L. "Multi-criteria group decision-making based on interval neutrosophic uncertain linguistic variables and Choquet integral", Cognitive Computation, 8(6), pp. 1036-1056 (2016).

8. Xu, Z.S. "Intuitionistic fuzzy aggregation operators", IEEE Transactions on Fuzzy Systems, 15(6), pp. 11791187 (2007).

9. Zadeh, L.A. "The concept of a linguistic variable and its application to approximate reasoning-I", Inf Sci, 8(3), pp. 199-249 (1975).

10. Zadeh, L.A. "The concept of a linguistic variable and its application to approximate reasoning-II", Inf Sci, 8(4), pp. 301-357 (1975).

11. Zadeh, L.A. "The concept of a linguistic variable and its application to approximate reasoning-III", Inf Sci, 9(1), pp. 43-80 (1975).

12. Liu, P.D. and Jin, F. "Methods for aggregating intuitionistic uncertain linguistic variables and their application to group decision making", Inf Sci, 205, pp. 58-71 (2012).

13. Wei, G.W. "Interval valued hesitant fuzzy uncertain linguistic aggregation operators in multiple attribute decision making", International Journal of Machine Learning and Cybernetics, 7(6), pp. 1093-1114 (2016).

14. Lu, M. and Wei, G.W. "Models for multiple attribute decision making with dual hesitant fuzzy uncertain linguistic information", International Journal of Knowledge-Based and Intelligent Engineering Systems, 20(4), pp. 217-227 (2016).

15. Li, Q.X., Zhao, X.F., and Wei, G.W. "Model for software quality evaluation with hesitant fuzzy uncertain linguistic information", Journal of Intelligent and Fuzzy Systems, 26(6), pp. 2639-2647 (2014).

16. Zhou, L.Y., Lin, R., Zhao, X.F., and Wei, G.W. "Uncertain linguistic prioritized aggregation operators and their application to multiple attribute group decision making", International Journal of Uncertainty, Fuzziness and Knowledge-Based Systems, 21(4), pp. 603-627 (2013).

17. Yu, X.H., Xu, Z.S., Liu, S.S., and Chen, Q. "Multicriteria decision making with two-dimension linguistic aggregation techniques", Int. J. Intell. Syst, 27(6), pp. 539-562 (2012).

18. Zhu, W.D., Zhou, G.Z., and Yang, S.L. "An approach to group decision making based on two-dimension linguistic assessment information", Syst. Eng, 27(2), pp. 113-118 (2009).

19. Liu, P.D. and Zhang, X. "An approach to group decision making based on two-dimension uncertain linguistic assessment information", Technol. Econ. Develop. Econ, 18(3), pp. 424-437 (2012).
20. Liu, P.D. and Yu, X.C. "Two-dimension uncertain linguistic power generalized weighted aggregation operator and its application for multiple attribute group decision making", Knowledge-Based Systems, 57(1), pp. 69-80 (2014).

21. Liu, P.D., Liu, Z.M., and Zhang, X. "Some intuitionistic uncertain linguistic Heronian mean operators and their application to group decision making", Applied Mathematics and Computation, 230(1), pp. 570-586 (2014).

22. Liu, P.D. and Wang, Y.M. "Multiple attribute decision-making method based on single valued neutrosophic normalized weighted Bonferroni mean", Neural Computing and Applications, 25(7-8), pp. 2001-2010 (2014).

23. Zhang, W.C., Xu, Y.J., and Wang, H.M. "A consensus reaching model for two-tuple linguistic multiple attribute group decision making with incomplete weight information", International Journal of Systems Science, 47(2), pp. 389-405 (2016).

24. Ji, P., Wang, J., and Zhang, H. "Frank prioritized Bonferroni mean operator with single-valued neutrosophic sets and its application in selecting third party logistics", Neural Computing and Applications, 30(3), pp. 799-823 (2018).

25. Nie, R.X., Wang, J., and Li, L. "2-tuple linguistic intuitionistic preference relation and its application in sustainable location planning voting system", Journal of Intelligent \& Fuzzy Systems, 33(2), pp. 885-899 (2017).

26. Peng, H., Wang, J., and Chen, P. "A linguistic intuitionistic multi-criteria decision-making method based on the Frank Heronian mean operator and its application in evaluating coal mine safety", International Journal of Machine Learning and Cybernetics, 9(6), pp. 1053-1068 (2018).

27. Peng, J.J., Wang, J., Wu, X., and Tian, C. "Hesitant intuitionistic fuzzy aggregation operators based on the archimedean t-norms and t-conorms", International Journal of Fuzzy Systems, 19(3), pp. 702-714 (2017).

28. Wang, J.Q., Yang, Y., and Li, L. "Multi-criteria decision-making method based on single valued neutrosophic linguistic Maclaurin symmetric mean operators", Neural Computing and Applications, 30(5), pp. 1529-1547 (2018).

29. Jiang, X.P. and Wei, G.W. "Some Bonferroni mean operators with two-tuple linguistic information and their application to multiple attribute decision making", Journal of Intelligent and Fuzzy Systems, 27, pp. 2153-2162 (2014).

30. Liu, P.D. "Multi-attribute decision-making method research based on interval vague set and TOPSIS method", Technological and Economic Development of Economy, 15(3), pp. 453-463 (2009).

31. Liu, P.D. and Wang, M.H. "An extended VIKOR method for multiple attribute group decision making based on generalized interval-valued trapezoidal fuzzy 
numbers", Scientific Research and Essays, 6(4), pp. 766-776 (2011).

32. Liu, P.D. and Zhang, X. "Research on the supplier selection of supply chain based on entropy weight and improved ELECTRE-III method", International Journal of Production Research, 49(3), pp. 637-646 (2011).

33. Yu, S.M., Wang, J., and Wang, J.Q. "An extended TODIM approach with intuitionistic linguistic numbers", International Transactions in Operational Research, 25(3), pp. 781-805 (2018).

34. Tian, Z.P., Wang, J., Wang, J.Q., and Zhang, H.Y. "An improved MULTIMOORA approach for multicriteria decision-making based on interdependent inputs of simplified neutrosophic linguistic information", Neural Computing and Applications, 28(sup 1), pp. 585-597 (2017).

35. Xu, Z.S. and Yager, R.R. "Some geometric aggregation operators based on intuitionistic fuzzy sets", International Journal of General Systems, 35(4), pp. 417-433 (2006).

36. Zhang, X., Liu, P.D., and Wang, Y.M. "Multiple attribute group decision making methods based on intuitionistic fuzzy frank power aggregation operators", Journal of Intelligent \& Fuzzy Systems, 29(5), pp. 2235-2246 (2015).

37. Wang, F., Zeng, S., and Zhang, C. "A method based on intuitionistic fuzzy dependent aggregation operators for supplier selection", Mathematical Problems in Engineering, 2013, pp. 1-9 (2013).

38. Meng, F., Zhang, Q., and Zhan, J. "The intervalvalued intuitionistic fuzzy geometric choquet aggregation operator based on the generalized banzhaf index and two-additive measure", Technological and Economic Development of Economy, 21(2), pp. 186215 (2015).

39. Bonferroni, C. "Sulle medie multiple di potenze", Bolletino Matematica Italiana., 5(3-4), pp. 267-270 (1950).

40. Wei, G.W., Zhao, X.F., and Lin, R. "Uncertain linguistic Bonferroni mean operators and their application to multiple attribute decision making", Applied Mathematical Modelling, 37(7), pp. 5277-5285 (2013).

41. Liu, P.D., Liu, J.L., and Chen, S.M. "Some intuitionistic fuzzy Dombi Bonferroni mean operators and their application to multi-attribute group decision making", Journal of the Operational Research Society, DOI: 10.1057/s41274-017-0190-y (2017).
42. Ding, Z.H. and Wu, Y.Y. "An improved intervalvalued hesitant fuzzy multi-criteria group decisionmaking method and applications", Mathematical and Computational Applications, 21(2), pp. 1-12 (2016).

43. Sun, H. and Sun, M. "Generalized Bonferroni harmonic mean operators and their application to multiple attribute decision making", Journal of Computational Information Systems, 8(14), pp. 5717-5724 (2012).

44. Xu, Z.S. "Induced uncertain linguistic OWA operators applied to group decision making", Inf Fusion, 7(2), pp. 231-238 (2006).

45. Xu, Z.S. "Fuzzy harmonic mean operators", International Journal of Intelligent Systems, 24(2), pp. 152$172(2009)$.

46. Liu, P.D. and Chu, Y.C. "Some two-dimensional uncertain linguistic Heronian mean operators and their application in multiple-attribute decision making", Neural Compute \& Application, 26(6), pp. 1461-1480 (2015).

\section{Biographies}

Peide Liu received the BS and MS degrees in Signal and Information Processing from Southeast University, Nanjing, China in 1988 and 1991, respectively. He obtained the $\mathrm{PhD}$ degree in Information Management from Beijng Jiaotong University, Beijing, China in 2010 .

He is currently a Professor with the School of Management Science and Engineering, Shandong University of Finance and Economics, Shandong, China. $\mathrm{He}$ is an Associate Editor of the Journal of Intelligent and Fuzzy Systems, the editorial board of the Journal Technological and Economic Development of Economy, and the members of editorial board of other 12 journals. He has authored or coauthored more than 130 publications. His research interests include aggregation operators, fuzzy logic, fuzzy decision-making, and their applications.

Weiqiao Liu received the BS in Management Sciences, Qufu Normal University, Rizhao 276826, Shandong Province, China in 2015. Now, she is studying for Master degree at the School of Management Science and Engineering, Shandong University of Finance and Economics, Jinan 250014, Shandong Province, China. Her research interests include aggregation operators, fuzzy logic, fuzzy decision-making, and their applications. 